\title{
Threshold for the destabilisation of the ion-temperature-gradient mode in magnetically confined toroidal plasmas
}

\author{
A. Zocco ${ }^{1,2, \dagger, ~ P . ~ X a n t h o p o u l o s ~}{ }^{1}$, H. Doerk ${ }^{2}$, J. W. Connor ${ }^{3}$ and P. Helander ${ }^{1}$ \\ ${ }^{1}$ Max-Planck-Institut für Plasmaphysik, D-17491, Greifswald, Germany \\ ${ }^{2}$ Max-Planck-Institut für Plasmaphysik, 85748, Garching, Germany \\ ${ }^{3}$ Culham Science Centre, Abingdon, Oxon OX14 3DB, UK
}

(Received 20 July 2017; revised 4 December 2017; accepted 6 December 2017)

The threshold for the resonant destabilisation of ion-temperature-gradient (ITG) driven instabilities that render the modes ubiquitous in both tokamaks and stellarators is investigated. We discover remarkably similar results for both confinement concepts if care is taken in the analysis of the effect of the global shear $\hat{s}$. We revisit, analytically and by means of gyrokinetic simulations, accepted tokamak results and discover inadequacies of some aspects of their theoretical interpretation. In particular, for standard tokamak configurations, we find that global shear effects on the critical gradient cannot be attributed to the wave-particle resonance destabilising mechanism of Hahm \& Tang (Phys. Plasmas, vol. 1, 1989, pp. 1185-1192), but are consistent with a stabilising contribution predicted by Biglari et al. (Phys. Plasmas, vol. 1, 1989, pp. 109-118). Extensive analytical and numerical investigations show that virtually no previous tokamak theoretical predictions capture the temperature dependence of the mode frequency at marginality, thus leading to incorrect instability thresholds. In the asymptotic limit $\hat{s} \iota \ll 1$, where $\iota$ is the rotational transform, and such a threshold should be solely determined by the resonant toroidal branch of the ITG mode, we discover a family of unstable solutions below the previously known threshold of instability. This is true for a tokamak case described by a local $\hat{s}-\alpha$ local equilibrium, and for the stellarator Wendelstein 7-X, where these unstable solutions are present even for configurations with a small trapped-particle population. We conjecture they are of the Floquet type and derive their properties from the Fourier analysis of toroidal drift modes of Connor \& Taylor (Phys. Fluids, vol. 30, 1987, pp. 3180-3185), and to Hill's theory of the motion of the lunar perigee (Acta Math., vol. 8, 1886, pp. 1-36). The temperature dependence of the newly determined threshold is given for both confinement concepts. In the first case, the new temperature-gradient threshold is found to be rather insensitive to the temperature ratio $T_{i} / T_{e}$, at least for $T_{i} / T_{e} \lesssim 1$, and to be a growing function of the density gradient scale for $T_{i} / T_{e} \gtrsim 1$. For Wendelstein $7-\mathrm{X}$, the new critical temperature gradient is a growing function of the temperature ratio. The importance of these findings for the assessment of turbulence in stellarators and low-shear tokamak configurations is discussed.

Key words: fusion plasma, plasma confinement, plasma instabilities

$†$ Email address for correspondence: alessandro.zocco@ipp.mpg.de 


\section{Introduction}

Any magnetic fusion reactor, no matter how carefully designed, is affected by turbulent heat losses, which are detrimental to its performance. The study of turbulent transport processes in magnetically confined plasmas, therefore, has always been of vital importance in the identification of the confinement properties of such devices. A number of plasma quantities are of relevance when evaluating how well the plasma is confined and thus more likely to yield fusion power. Amongst many, there is no doubt that one of the most important is the achievable plasma temperature gradient.

It is known that kinetic plasma instabilities can draw energy from equilibrium plasma gradients and the resulting turbulence cause what is observed as an anomalous energy loss. Perhaps the most important of such instabilities is the ion-temperature-gradient (ITG) driven one, which has been extensively studied both in tokamaks and stellarators. The ITG mode manifests itself in several flavours. Sometimes it is a modified unstable sound wave, as derived for the first time by Rudakov \& Sagdeev (1961); it can also arise in the guise of the interchange-type mode of Rosenbluth \& Longmire (1956) as a result of equilibrium magnetic field gradients (Horton, Choi \& Tang 1981). It can be of the ballooning type (Connor \& Taylor 1987), with a broad radial structure (Romanelli \& Zonca 1993); of the 'boxing' type (Plunk et al. 2014), or with detailed structure on equilibrium magnetic surfaces (Zocco et al. 2016); of the isolated or general (Dickinson et al. 2014) type, or else 'passing' or 'trapped' (Dewar 1997); this list may not be complete.

In all these cases, the instability can be categorised in two broad classes which correspond to the slab and the toroidal branch of the mode. In the first case, instability occurs when the equilibrium temperature gradient causes a change in temperature of a perturbation propagating at the sound speed, and such change is in phase with a perturbed $\boldsymbol{E} \times \boldsymbol{B}$ drift, also caused by the presence of the equilibrium temperature gradient (see discussion after (16) of Coppi, Rosenbluth \& Sagdeev (1967)). In toroidal geometry, the coupling of the $\nabla B-$ and curvature drifts with the equilibrium temperature gradient can also destabilise the mode (Horton et al. 1981). The intuitive picture of the ITG destabilisation generally holds for very large temperature gradients (fluid limit). However, in many operationally relevant regimes (Mantica et al. 2009), temperature gradients are not so extreme as to justify a fluid approximation, and it is necessary to account for resonances. The complications associated with such theories escalate very rapidly, and an intuitive picture of the kinetic resonant destabilisation would perhaps be more confusing rather than clarifying. The development of gyrokinetic numerical codes has rescued the situation, and has advanced our understanding of the critical threshold for the destabilisation of the ITG mode. ${ }^{1}$ Inevitably, the interpretation of any linear result produced by a gyrokinetic code is ultimately based on fundamental works, most notably those of Terry, Anderson \& Horton (1982), Biglari, Diamond \& Rosenbluth (1989) and Romanelli (1989) (hereafter BDR), for the toroidal branch of the instability, and those of Kadomtsev \& Pogutse (1970), and Hahm \& Tang (1989) for the finite-shear and shear-less slab branches, respectively.

All these analytical theories of the resonant destabilisation of the toroidal ITG were performed in a long-wavelength limit, thus neglecting the full ion Larmor radius response. Here, we present, for the first time, a complete mathematical treatment where this response is retained. The analysis is performed in the local kinetic limit,

\footnotetext{
${ }^{1}$ In this context, a very influential work is that of Jenko, Dorland and Hammett (Jenko, Dorland \& Hammett 2001).
} 
thus neglecting the variation of the eigenfunction along the magnetic field line, but is valid for arbitrary magnetic geometry. Analytical progress is made by introducing a Padé approximant for the ion response after integrating over the resonances. These are treated by using a new series representation of the Owen $T$-function (Owen 1956) which exploits the properties of the incomplete Euler gamma function (Tricomi $1950 b, a)$. No approximation for the velocity-space dependence of the particles drifts is made.

The threshold for resonant destabilisation of the toroidal branch of the ITG mode is then calculated and compared to previous analytical results and to numerical results obtained from simulations performed with the GENE (Jenko et al. 2000) code for a local $\hat{s}-\alpha$ tokamak equilibrium (Connor, Hastie \& Taylor 1978). In the limit in which the streaming term contribution is negligible $)^{2}(\hat{s} \ll 1)$, we identify a family of unstable ion modes below the threshold for destabilisation of the toroidal branch of the ITG mode. Such modes require high velocity-space resolution to be adequately resolved, they are more unstable for small inverse aspect ratios, and do not show resonant structures for $v_{\|} \neq 0$. The new critical threshold is studied, for several density gradients, as a function of $\tau=T_{i} / T_{e}$, where $T_{i}$ and $T_{e}$ are the ion and electron temperature, respectively. For $\tau \gtrsim 1$, the threshold shows a dependence on $\tau$. For $\tau \lesssim 1$, the threshold in the inverse temperature-gradient scale $R / L_{T}$ is proportional to the inverse density gradient scale, $R / L_{n}$.

The same qualitative small- $\hat{s}$ behaviour at marginal stability is observed in the stellarator Wendelstein 7-X, even for flat densities. We have verified that the modes are present for configurations with a small trapped-particle population. From the common features shown in the tokamak and stellarator cases, we argue that these modes are of the Floquet type, and can be described in terms of Mathieu functions. Far from marginality, we propose a general eigenvalue equation that is exact and based on Hill's mathematical study of the motion of the Lunar perigee (Hill 1886).

The article is organised as follows. In $\S 2$ we present the numerical results from gyrokinetic simulations that demonstrate the qualitative similar behaviour of low- $\hat{s}$ tokamaks and Wendelstein7 $-X$ at marginal stability. In $\S 3$ the critical threshold for the low-shear ITG mode is shown for both the $\hat{s}-\alpha$ tokamak equilibrium and Wendelstein 7-X. The exact analytical formula for Floquet modes is given (and solved) in $\S 4$. Conclusions are given in $\S 5$. A series of appendices is attached to elucidate some analytical aspects of the resonant destabilisation of the toroidal ITG.

\section{Instability thresholds}

The description of the resonant destabilisation of the toroidal ITG is based on the following eigenvalue equation (Terry et al. 1982; Biglari et al. 1989; Romanelli 1989)

$$
1+\tau=I_{i} \equiv \int \mathrm{d}^{3} \boldsymbol{v} \frac{\omega-\omega_{* i}^{T}}{\omega-\omega_{\mathrm{di}}} J_{0}^{2}\left(\boldsymbol{k}_{\perp}^{2} \rho_{i}^{2} \hat{v}_{\perp}^{2}\right) \frac{F_{0 i}}{n_{0}},
$$

where $\tau=T_{i} / T_{e}, \omega$ is the mode complex frequency, $\boldsymbol{k}_{\perp}$ is the wave vector perpendicular to the equilibrium magnetic field, $\hat{v}^{2}=\left(v_{\|}^{2}+v_{\perp}^{2}\right) / v_{\text {thi }}^{2}$, with $v_{\text {thi }}=\sqrt{2 T_{i} / m_{i}}$ the ion thermal speed and $m_{i}$ and $n_{0}$ are the ion mass and density, respectively. We are effectively considering a local limit, in the sense that the eigenmode structure along the field-following coordinate is neglected, and we restrict our attention to a particular location along the equilibrium magnetic field. This is equivalent to

\footnotetext{
${ }^{2}$ While this is the case in tokamaks, in a stellarator this is not the only relevant condition.
} 
completely neglecting the slab branch discussed in the introduction, and taking $k_{\|} v_{\text {thi }} \ll \omega$, where $k_{\|}$is the parallel wavenumber. The equilibrium distribution function is taken to be Maxwellian,

$$
F_{0 i}=\frac{n_{0}}{\left(\pi v_{\text {thi }}^{2}\right)^{3 / 2}} \mathrm{e}^{-\hat{v}^{2}} .
$$

Two characteristic frequencies are present in (2.1)

$$
\omega_{* i}^{T}=\omega_{* i}\left[1+\eta_{i}\left(\hat{v}^{2}-3 / 2\right)\right],
$$

with $\omega_{* i}=0.5 k_{y} \rho_{i} v_{\text {thi }} / L_{n}, \eta_{i}=L_{n} / L_{T}$, and

$$
\omega_{d}=\left(\omega_{\kappa} \hat{v}_{\|}^{2}+\omega_{B} \hat{v}_{\perp}^{2} / 2\right),
$$

where $\rho_{i}=v_{\text {thi }} / \Omega_{c i}$ is the ion Larmor radius, $\Omega_{c i}=e B /\left(m_{i} c\right)$ the ion cyclotron frequency, $\omega_{\kappa}=0.5 k_{y} \rho_{i} v_{\text {thi }} / a$ and $\omega_{B}=\omega_{\kappa}-\mathrm{d} \beta / \mathrm{d} x$ the magnetic curvature and $\nabla B$-drift frequencies, while $L_{n}^{-1}=-n_{0}^{-1} \mathrm{~d} n_{0} / \mathrm{d} x$ and $L_{T}^{-1}=-T_{i}^{-1} \mathrm{~d} T_{i} / \mathrm{d} x$ define the characteristic density and temperature-gradient scales, and $\beta=8 \pi n_{0}\left(T_{e}+T_{i}\right) / B^{2}$ is the ratio of kinetic to magnetic pressure (which will shortly be set to zero), $x$ is the radial coordinate and $y$ is a coordinate associated with the field-line labels on a magnetic surface. Notice that we are assuming $k_{x}=0$ for simplicity. The Bessel function squared originates, as usual, from a Fourier transform of the gyroaveraged potential followed by a $\mathrm{d}^{3} \boldsymbol{v}$ integral at constant $\boldsymbol{r}$, the particle position. In (2.1) the eigenvalue $\omega$ acquires non-zero imaginary values that regularise the, otherwise, singular integrand. The analytical form of the integral $I_{i}$ for arbitrary Larmor radii is given in appendix A. The drift-kinetic limit is sufficient for the following discussion. In this case $\boldsymbol{k}_{\perp} \rho_{i} \rightarrow 0, J_{0}^{2} \rightarrow 1$, and it is easy to show that

$$
\begin{aligned}
D \equiv & -(1+\tau)+I_{i} \\
= & -(1+\tau)+\left(1-\frac{\omega_{* i}}{\omega}\right) \frac{\omega}{\omega_{\kappa}} Z^{2}\left(\sqrt{\frac{\omega}{\omega_{\kappa}}}\right) \\
& +\eta_{i} \frac{\omega_{* i}}{\omega}\left\{\left(1-2 \frac{\omega}{\omega_{\kappa}}\right) \frac{\omega}{\omega_{\kappa}} Z^{2}\left(\sqrt{\frac{\omega}{\omega_{\kappa}}}\right)-2\left(\frac{\omega}{\omega_{\kappa}}\right)^{3 / 2} Z\left(\sqrt{\frac{\omega}{\omega_{\kappa}}}\right)\right\},
\end{aligned}
$$

which corresponds to $D_{0}$ in (3) of BDR (Biglari et al. 1989). In the appendices we prove this result in two different ways; both are alternatives to the derivation of Biglari et al. (1989). The real frequency at marginality is evaluated by solving $\operatorname{Im}\left[D\left(\omega_{r}\right)\right] \equiv$ $D_{i}\left(\omega_{r}\right)=0$, where one sets $\omega=\omega_{r}+\mathrm{i} \gamma$, and takes $\gamma \rightarrow 0$. Using (2.5) this means

$$
\left\{1-\frac{\omega_{* i}}{\omega_{r}}+\eta_{i} \frac{\omega_{* i}}{\omega_{r}}\left(1-2 \frac{\omega_{r}}{\omega_{\kappa}}\right)\right\} \operatorname{Re}\left[Z\left(\sqrt{\frac{\omega_{r}}{\omega_{\kappa}}}\right)\right]=\eta_{i} \frac{\omega_{* i}}{\sqrt{\omega_{r} \omega_{\kappa}}} .
$$

Once $\omega_{r}$ is found, the condition that determines destabilisation is $\operatorname{Re}\left[D\left(\omega_{r}\right)\right]>0$, which can be determined only numerically. However, Romanelli (Romanelli 1989) has evaluated the integral $I_{i}$ by replacing $v_{\perp}^{2} / 2+v_{\|}^{2} \rightarrow 4 / 3\left(v_{\perp}^{2}+v_{\|}^{2}\right)$ in the definition of the particle magnetic drifts (see (2.4)) in order to fit the numerical solution of (2.1) at $R / L_{n} \ll 1$, for $k_{y} \rho_{s}=\sqrt{0.1} \approx 0.32$, where $\rho_{s}=\sqrt{T_{i} / m_{i}} / \Omega_{i}=\rho_{i} / \sqrt{2}$. In his case, the condition $\operatorname{Re}\left[D\left(\omega_{r}\right)\right]>0$ yields a critical gradient $R /\left.L_{T}\right|_{\text {crit }}=(1+\tau)(4 / 3)$ whose $\tau$ dependence comes from the obvious fact that $\operatorname{Re}\left[D\left(\omega_{r}\right)\right]>0$ implies 


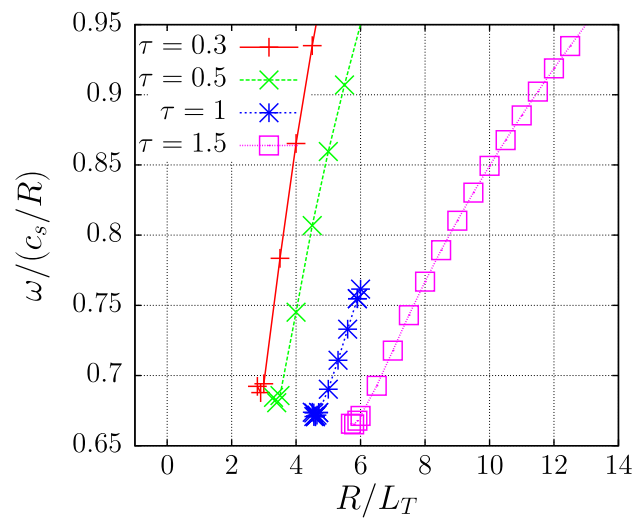

FIGURE 1. Real frequency from the GENE code as a function of the temperature gradient for different $\tau=T_{i} / T_{e}$. The $\tau$ dependence is evident. Cyclone base case parameters are used (see text for details).

$\operatorname{Re}\left[I_{i}\left(\omega=\omega_{r}\right)\right]>1+\tau$. Obtaining the correct mode frequency at marginality is therefore crucial. From direct numerical simulations we see that this quantity shows a dependence on the ion temperature that is not captured by Romanelli's treatment, nor by any other analytical theory known to us (see figure 1).

In our numerical tests, we consider adiabatic electrons and typical cyclone base case (CBC) parameters for $\hat{s}-\alpha$ geometry: $\hat{s}=0.786, q=\iota^{-1}=1.4, \epsilon=r / R=0.18$, $R / L_{n}=2, k_{y} \rho_{s}=0.3$, where $R$ is the device major radius. From the imaginary part of the eigenvalue, we extract the critical gradient (see figure 2). Figure 3 shows the resulting critical gradient compared to several theories and one fitting formula. The linear fit of the numerical data gives ${ }^{3} R /\left.L_{T}\right|_{\text {crit }}=(2.45 \pm 0.07) \tau+2.07 \pm 0.06$, with a reduced $\chi^{2}=6.5 \times 10^{-4}$. The fitting formula of Jenko et al. (2001) (hereafter denoted by JDH) is $R /\left.L_{T}\right|_{\text {crit }}=(1+\tau)(4 / 3+1.91 \hat{s} / q)$. The curve derived by Romanelli (1989), $R / L_{T}=(1+\tau)(4 / 3)$, which should work better for flat densities, $R / L_{n} \rightarrow 0$, is not expected to match the numerical results for $R / L_{n}=2, R / L_{T}=O(1)$, even if $q \rightarrow \infty$ (in which case the streaming term effect measured by the factor $\hat{s} / q$ in the JDH formula should be negligible, as it is neglected in (2.1)). The BDR condition for destabilisation (Biglari et al. 1989) would give $R / L_{T}=2.7$, with virtually no dependence on $\tau$. The full ion Larmor radius resonant theory developed in the present article predicts higher critical gradients than the long-wavelength resonant theory. Like results from numerical simulations, it gives a critical gradient that depends linearly on $\tau=T_{i} / T_{e}$, but it underestimates the numerical results. As already mentioned, this discrepancy can be attributed to the fact that analytical theories predict a real frequency at marginality that does not depend on $\tau$, as opposed to the numerical findings. This is true for all the analytical theories revisited and derived here, and seems to be a consequence of the neglect in (2.1) of the streaming term. The problem is, of course, that parallel streaming has been neglected in the derivation of (2.1), which can only be satisfied at a single (or a pair of) poloidal location on the flux surface. Taken literally, this equation thus implies that the entire mode structure is concentrated at this point, which, in the analytical theories quoted, is the location where the field curvature is most unfavourable. However, in all practical circumstances, that is not what the mode

\footnotetext{
${ }^{3}$ We warn the reader that $R$ here is representative of the curvature of the magnetic field. In a stellarator, the natural normalisation scale of the temperature-gradient length is not the major radius of the machine.
} 


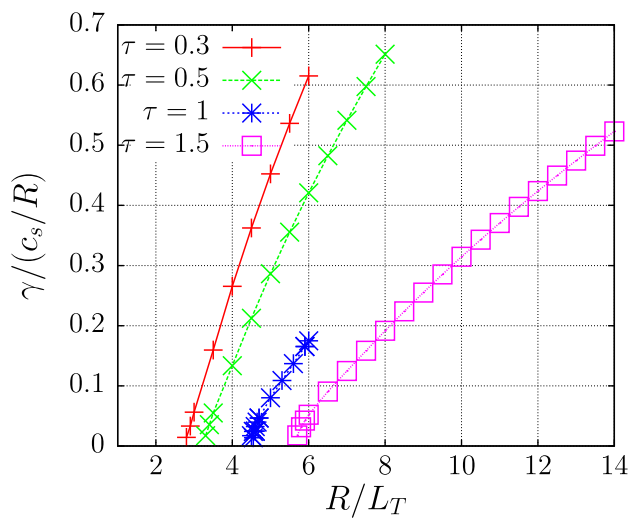

FIGURE 2. Growth rate from the GENE code as a function of the temperature gradient for different $\tau=T_{i} / T_{e}$. The $\tau$ dependence is evident. CBC parameters are used (see text for details).

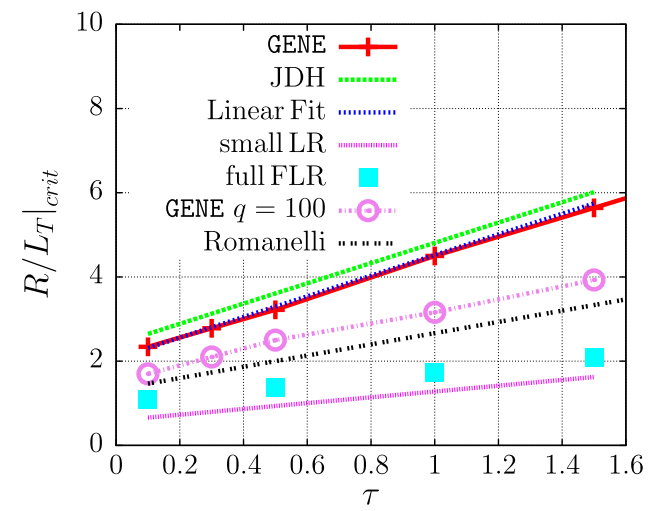

FIgURE 3. Critical threshold from GENE data, linear fit to GENE data, fitting formula of Jenko Dorland and Hammett and different local theories. The GENE data at $q=100$ should be compared to the local theories.

structure is, and the streaming term (with its $\partial_{\theta}$ derivatives, where $\theta$ is the ballooning angle) determines the rate at which the eigenmode decays along the field line.

The results from GENE simulations are somewhat consistent with the fitting formula of Jenko et al. (2001) (hereafter JDH) but do not seem to relate to the theory of Hahm \& Tang (1989), since we find a stabilising contribution from finite shear. This is in line with a statement present in the work of Biglari et al. (1989). ${ }^{4}$ The remaining two curves are derived from the numerical solution of the dispersion relation with full Larmor orbits, equation (A 1), for small $\left(\boldsymbol{k}_{\perp} \rho_{i} \ll 1\right)$ and for arbitrary Larmor

\footnotetext{
${ }^{4}$ The stability diagram of figure 5 of Biglari et al. (1989) has also been reproduced (not shown) by solving (2.5). Their result, $R / L_{T}=2.7$, can be obtained only if we include an extra multiplicative factor in the definition of the magnetic drift, e.g. $\omega_{\kappa}=2 \omega_{* i}\left(L_{n} / R\right)$ (however, in this work we follow Hastie \& Hesketh (1981) and use $\omega_{\kappa}=\omega_{* i}\left(L_{n} / R\right)$ ), and the frequency at marginal stability is evaluated by solving the equation
}

$$
\left\{\left[1-\frac{\omega_{* i}}{\omega_{r}}+\eta_{i} \frac{\omega_{* i}}{\omega_{r}}\left(1-2 \frac{\omega_{r}}{\omega_{\kappa}}\right)\right] \operatorname{Re}\left[Z\left(\sqrt{\frac{\omega}{\omega_{\kappa}}}\right)\right]-\eta_{i} \frac{\omega_{* i}}{\sqrt{\omega_{r} \omega_{\kappa}}}\right\} \omega_{r}=0
$$




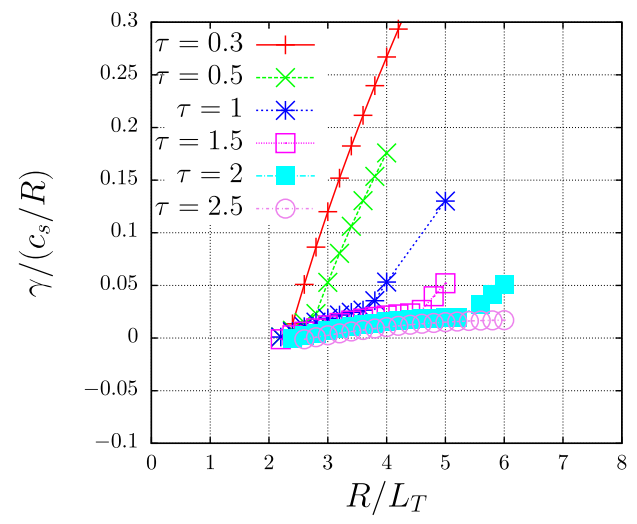

FIGURE 4. Imaginary part of eigenvalue from the GENE code as a function of the temperature gradient for different $\tau$. Parameters as in figure 1 , but $\hat{s}=0.1, \epsilon=0.03$. The 'background' modes at marginality are evident.

radii. Strictly speaking, all our numerical results are valid for one specific wavelength: $k_{y} \rho_{s}=0.3$. However, a dependence of the numerical results on $k_{y}$ cannot be excluded. A sensitivity scan was performed for the reference value $\tau=1$. If we vary $0.2 \leqslant k_{y} \rho_{s} \leqslant$ 0.4 , we find a critical gradient in the range $4.35 \leqslant R /\left.L_{T}\right|_{\text {crit }} \leqslant 4.8$ This indeterminacy is small enough to discriminate the analytical results of figure 3 , and large enough to let us consider the JDH formula quite reliable.

This preliminary analysis is in fact more general that it would seem. Equations (2.1) and (A 1) were derived by neglecting the contribution associated with particle streaming, but the magnetic drift frequencies $\omega_{\kappa}$ and $\omega_{B}$ are essentially arbitrary. They must therefore be applicable to the stellarator case as well. There is a key factor that now brings tokamaks and stellarators together. A low-bootstrap-current stellarator, such as Wendelstein 7-X, possesses a low global shear. This condition is, incidentally, the same condition that is required to neglect particle streaming in the kinetic equation, and the slab branch of the ITG instability (Jenko et al. 2001) for the tokamak case. Thus, for low shear, the resonant destabilisation of the ITG mode in tokamaks and stellarators could show some common features. In the following sections we will discover what these common aspects are.

\subsection{Low-shear tokamak}

We repeat the same study that was performed above to benchmark the analytical theories of the destabilisation of the toroidal ITG, but for $\hat{s}=0.1$, and $\epsilon=0.03$, instead of $\hat{s}=0.768$ and $\epsilon=0.18$. The results are shown in figures (4)-(5), and exhibit a new family of unstable modes below what would have been the critical gradient had we extrapolated to $\gamma \rightarrow 0$ the growth rate calculated at large $R / L_{T}$. These modes behave as a persistent 'background' of instability. The velocity-space structures and the eigenfunctions of two representative modes (one from the 'background', one from the strongly driven toroidal branch) are compared in figures 6 and 7. For these cases, a velocity-space resolution of $\mathrm{lv} / \mathrm{nv} 0=3 / 512 \approx 5.86 \times 10^{-3}$, where $\mathrm{lv}$ is the extension of the simulation box (in units of thermal speed), and nv0 is the number of grid points in the $v_{\|}$- direction, was required to fully resolve the maximum of the distribution function of the background mode at $v_{\|}=0$. However, the eigenvalue 


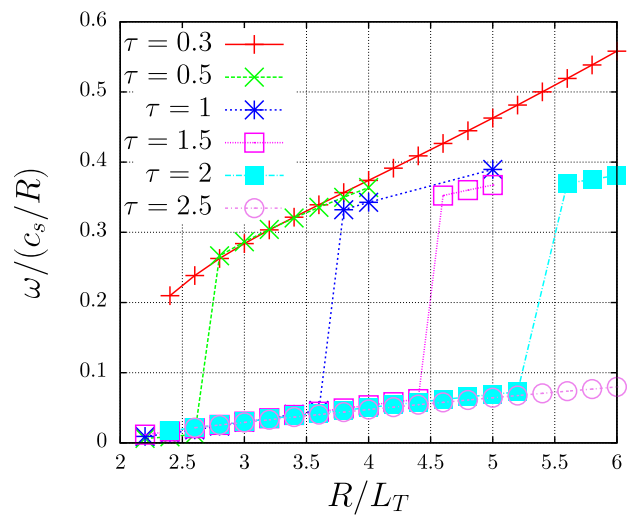

FIGURE 5. Real part of eigenvalue from the GENE code as a function of the temperature gradient for different $\tau$. Parameters as in figure 1 , but $\hat{s}=0.1, \epsilon=0.03$. The 'background' modes at marginality are evident.

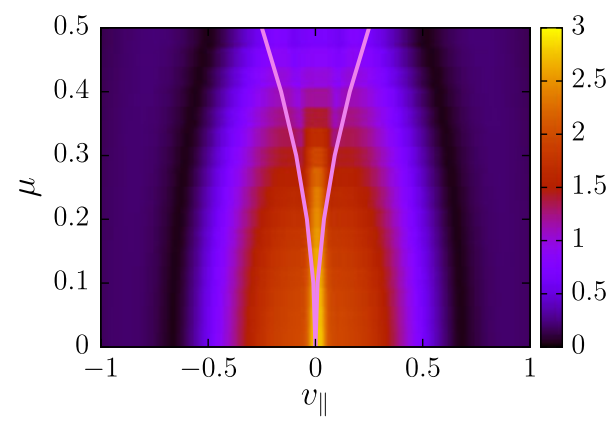

FIGURE 6. Ion distribution function in phase space. $\tau=1, R / L_{T}=3$, for the 'background' mode that belongs to the 'foot' of figure 5 . No clear resonance at finite $v_{\|}$. The velocityspace resolution in the $v_{\|}$-direction is $5.86 \times 10^{-3}$. Other parameters as in figure 5 .

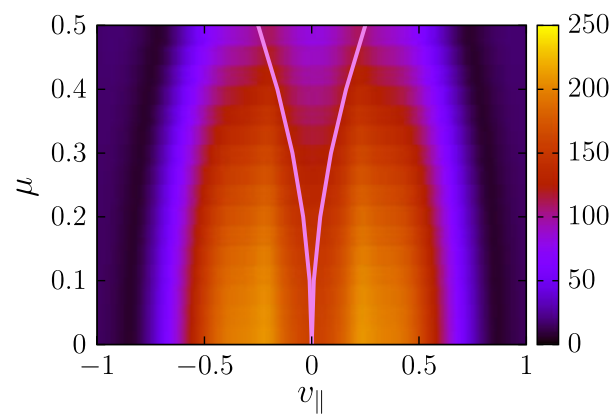

FIGURE 7. Ion distribution function in phase space. $\tau=1, R / L_{T}=5$, for the toroidal branch mode. Resonance occurs outside the $\mu \pm \hat{v}_{\|}^{2} \equiv$ const. boundary. The velocity-space resolution in the $v_{\|}$-direction is $5.86 \times 10^{-3}$. Other parameters as in figure 5 .

would not be affected by choosing slightly larger values. All other resolutions are kept consistent with the analysis of the previous section, but care must be taken to fully resolve the asymptotic behaviour of the 'background' mode eigenfunctions for 


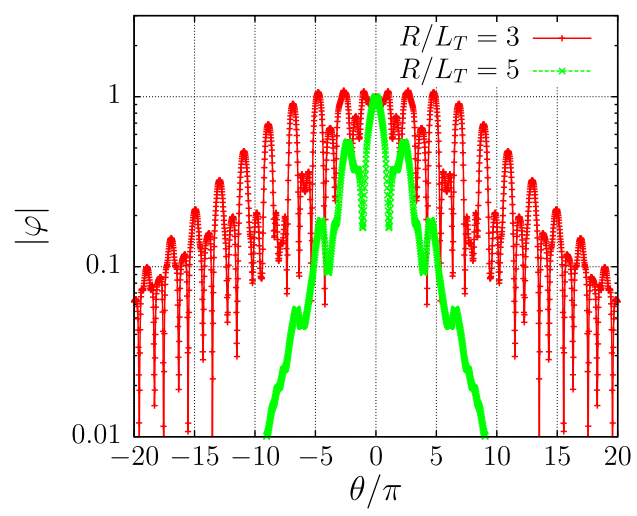

FIGURE 8. Eigenfunctions as a function of the ballooning angle for the $\hat{s}-\alpha$ tokamak equilibrium from the GENE code (flux tube). Here $\tau=1, k_{y} \rho_{s}=0.3, a / L_{T}=3$ (Floquet), $a / L_{T}=5$ (toroidal branch). The velocity-space resolution in the $v_{\|}$direction is $5.86 \times 10^{-3}$. Other parameters as in figure 4.

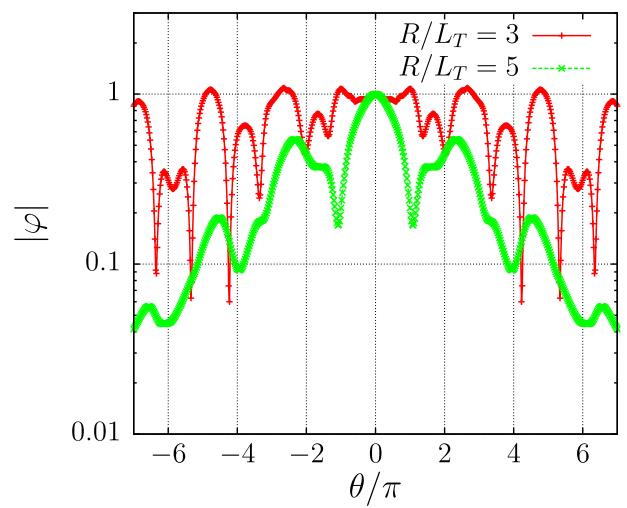

FIGURE 9. A 'zoom' of figure 8. Eigenfunctions as a function of the ballooning angle for the $\hat{s}-\alpha$ tokamak equilibrium from the GENE code (flux tube). Here $\tau=1, k_{y} \rho_{s}=0.3$, $a / L_{T}=3$ (Floquet), $a / L_{T}=5$ (toroidal branch). The velocity-space resolution in the $v_{\|}$ direction is $5.86 \times 10^{-3}$.

large ballooning angles. Since, formally, the ballooning angle is the Fourier conjugate of the local radial variable, asymptotically decaying eigenfunctions are captured by increasing the radial resolution. In our case, for a domain $L_{x}=1 /\left(k_{y} \rho_{s} \hat{s}\right) \approx 33.33$, the number of grid points in the radial $(x)$ direction varies from 24 to 64 . A comparison of the eigenfunctions for the two types of modes is in figures 8 and 9. From the first, we see that, at marginality, both eigenfunctions decay rather slowly. However, the decay length of the background mode is much greater. Furthermore, the background mode does not have an absolute maximum at $\theta=0$. While the strongly driven mode shows resonances at finite $v_{\|}$outside the $\mu \pm \hat{v}_{\|}^{2} \equiv$ const. boundary, the background mode does not show structure at finite $v_{\|}$. Its dependence on the inverse aspect ratio is shown in figure 10 . We see that the slab character of the instability is more pronounced for larger aspect ratios. It is of some interest to know whether, for a given aspect ratio, there is a critical shear, $\hat{s}_{\text {crit }}$, above which the 'background' 


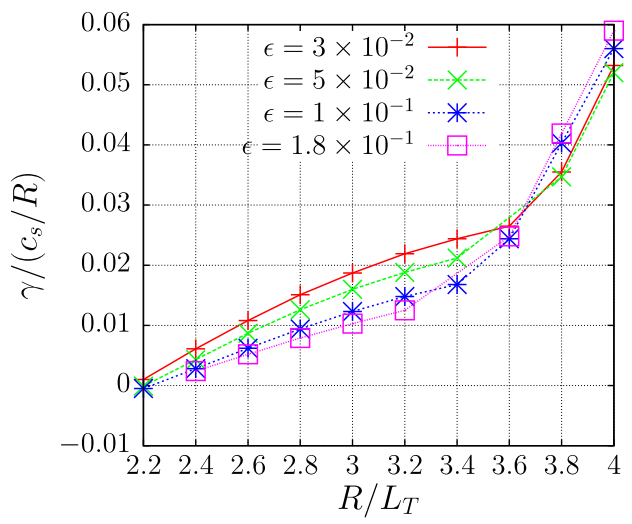

FIgURE 10. A 'zoom' of the low shear, $\hat{s}=0.1$, 'background' marginal modes for $\tau=1$ for several inverse aspect ratios.

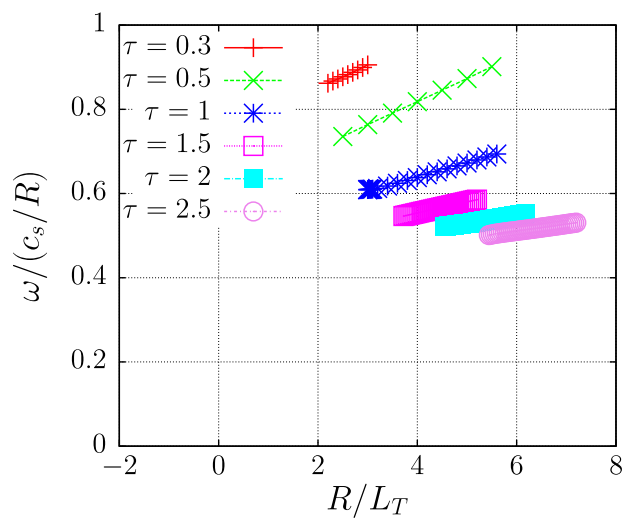

FIGURE 11. Real part of eigenvalue from the GENE code as a function of the temperature gradient for different $\tau$. Parameters as in figure 1 , but $\hat{s}=0.1$ and $R / L_{n}=0$. The $\tau$ dependence at marginality is evident.

modes are suppressed. For the case $R / L_{T}=3$, and $\epsilon=0.18$ of figure 10 , we find $0.14<\hat{S}_{\text {crit }}<0.16$. For flat density profiles, $R / L_{n}=0$, and moderate inverse aspect ratio, $\epsilon=0.18$, the real frequency at marginality shows an even stronger dependence on the temperature ratio, $\tau$, (figure 11) but, for the specific parameters used, the background modes are not present. The equilibrium values chosen to let the background modes emerge could seem unrealistic. Let us therefore consider a situation where such modes are always expected, almost by design.

\subsection{Wendelstein 7-X}

The stellarator Wendelstein 7-X shows the same qualitative behaviour as just described for the tokamak case. We consider a high-mirror configuration (Geiger et al. 2015) at a normalised radial position $r / a=0.7$, for $k_{y} \rho_{s}=0.9$, flat density $a / L_{n}=0, q=$ $1 / \iota=1.10186$ and small negative global shear $\hat{s}=-0.1286$. Here $a$ is the average minor radius. Such configuration can be considered to be 'standard'. It is realised by setting to zero the current in the planar coils, and imposing a 'large' ratio of 


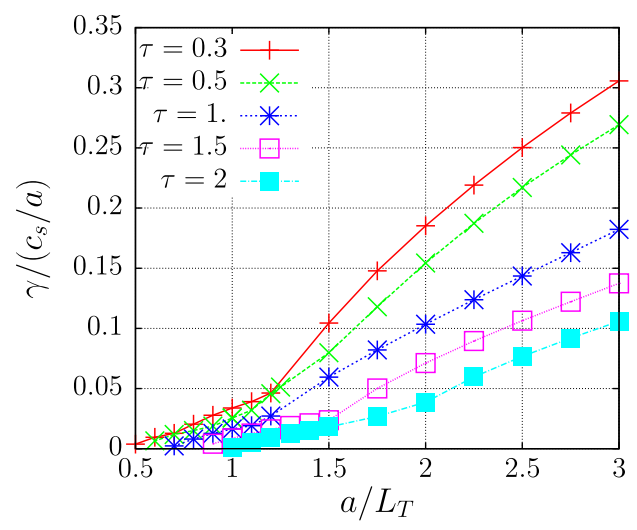

FIGURE 12. Growth rate of microinstability in Wendelstein 7-X from the GENE code (flux tube). High-mirror configuration, adiabatic electrons, radial position $r / a=0.7$. The 'background' modes at marginality are evident.

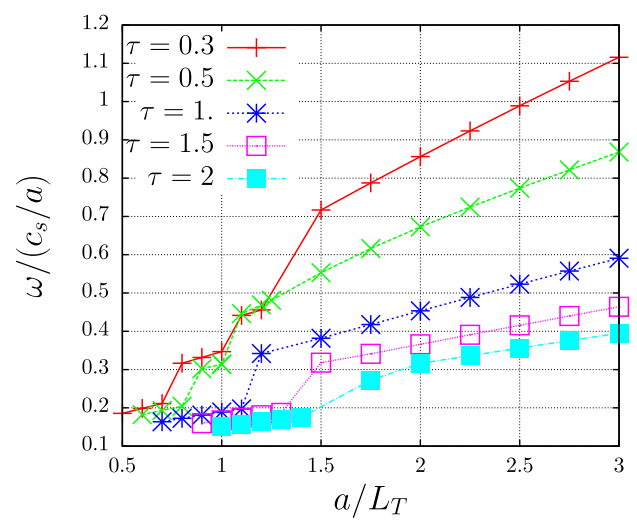

FIGURE 13. Frequency associated with the unstable modes of figure 12 evaluated for Wendelstein 7-X from the GENE code (flux tube). High-mirror configuration, adiabatic electrons, radial position $r / a=0.7$, for $k_{y} \rho_{s}=0.9$.

average toroidal to helical curvature, $b_{1,0} / b_{1,1} \approx 0.5$, and a finite-mirror term, $b_{0,1} \approx 0.1$, where $b_{n, m}$ are the Fourier components of the equilibrium magnetic field in Boozer coordinates (Xanthopoulos et al. 2009). A scan in normalised temperature-gradient scale, $a / L_{T}$, is given in figures 12 and 13 for several $\tau$. As in the tokamak case, there is a background of unstable modes below the threshold of the strongly driven toroidal ITG branch. An inspection of the real part of the eigenvalue reveals more frequent, but milder, jumps than in the tokamak case. The eigenfunction is plotted in figure 14 for $a / L_{T}=0.8$ (background) and $a / L_{T}=3$. (toroidal branch) for $\tau=1$. The global shear is kept constant, the mode localisation of the two modes is very different: the background mode shows a much slower variation along the field-line-following coordinate than the toroidal branch mode. Our results suggest that the linear threshold for destabilisation of ITG modes in Wendelstein 7-X could be overestimated by a rough extrapolation of the toroidal branch to $\gamma \rightarrow 0$, even for flat densities. 


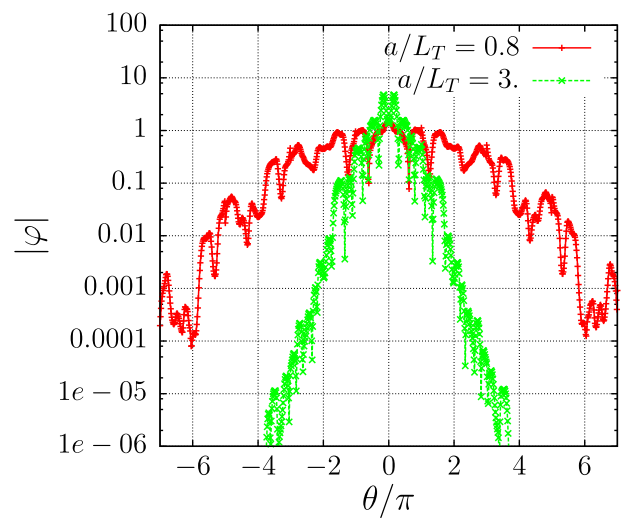

FIGURE 14. Eigenfunctions for Wendelstein 7-X from the GENE code (flux tube). Highmirror configuration, adiabatic electrons, radial position $r / a=0.7$. Here $\tau=1, k_{y} \rho_{s}=0.9$, $a / L_{T}=0.8$ (Floquet), $a / L_{T}=3$ (toroidal branch).

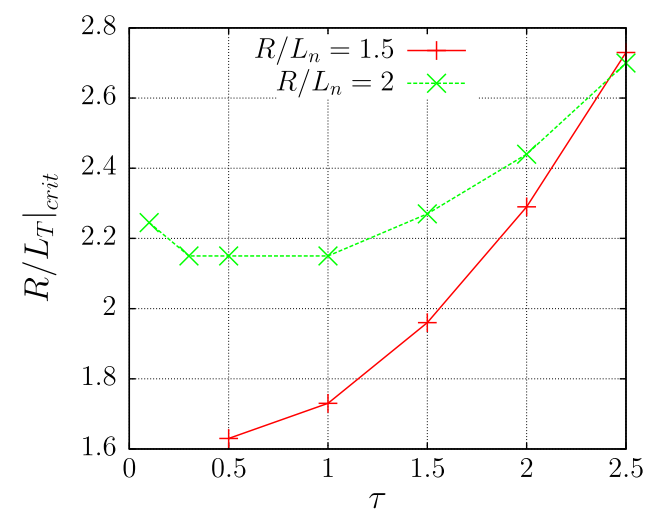

FIgURE 15. Critical threshold from GENE data of figure 5 for several density gradients, $R / L_{n}$.

\section{Temperature dependence of critical thresholds}

In the previous section we have conjectured that the resonant destabilisation of the ITG mode in low-shear tokamaks and stellarators possesses common features. Numerical simulations corroborate this hypothesis. We refrain from proposing some universal formula for critical gradients at low shear, nonetheless we find it instructive to explore the temperature dependence of the critical gradient, since this puts the new results in the same context as previous analytical studies.

In the asymptotic limit of large inverse aspect ratio, $\epsilon=0.03$, the $\hat{s}-\alpha$ tokamak case gives a critical inverse gradient that increases with $\tau$, for $\tau \gtrsim 1$. For $\tau \lesssim 1$, the instability threshold is fixed by the density gradient, e.g. $\left(R / L_{T}\right)_{\text {crit }} \approx R / L_{n}$ (see figure 15). In accordance with the JDH formula, a decreasing shear and an increasing $L_{n} / L_{T}$ have a destabilising effect (see figure 16). The results for Wendelstein $7-X$ (figure 17) were all derived in the flat density limit $R / L_{n}=0$. The critical temperature gradient is a growing function of $\tau$. We observe that $\left(a / L_{T}\right)_{\text {crit }} \approx 1$ for sufficiently hot ions, $\tau \gtrsim 2$. 


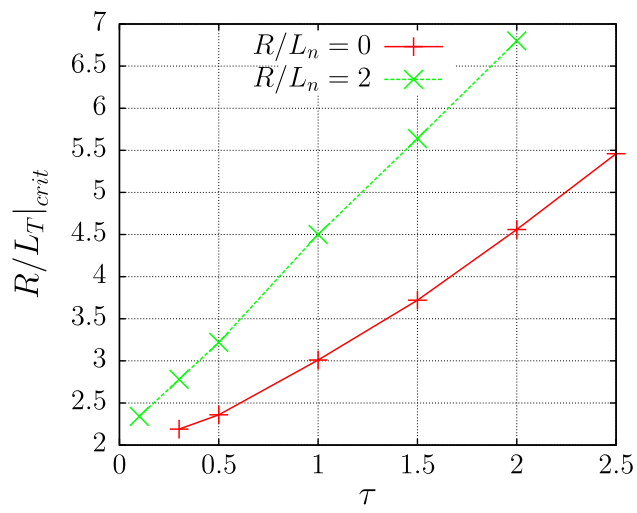

FIGURE 16. Critical threshold from GENE. CBC parameters are used (see text for details), however $\hat{s}=0.1$ for $R / L_{n}=0$ and $\hat{s}=0.786$ for $R / L_{n}=2$.

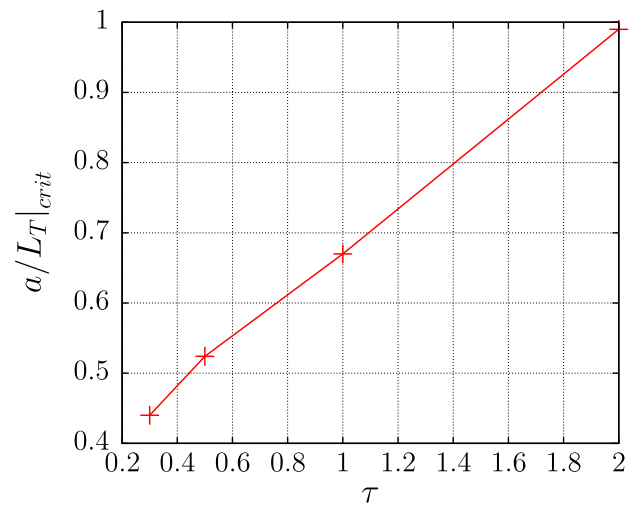

FIgURE 17. Critical gradient for Wendelstein $7-X$ as a function of the temperature ratio $\tau=T_{i} / T_{e}$.

\section{Floquet solutions}

What are the modes that emerge below the critical threshold of the toroidal branch at low shear? In the classical nomenclature of ITG modes in tokamaks, for $\hat{s} / q \approx$ $\iota \hat{s} \ll 1$, the slab branch of the instability tends to be negligible. Below marginality the toroidal branch is stable. We saw that the background of modes at marginality is not eliminated at large inverse aspect ratios in tokamaks. High-mirror, as well as low-mirror, Wendelstein 7-X configurations also exhibit this persistent feature, and we recall that density gradients are stabilising since the electrons are taken to be adiabatic. To shed some light on the nature of these modes, we are compelled to abandon for a moment the regime of marginality and consider these modes at their maximum growth rate. We first verify where this maximum is. We select two representative cases for $\epsilon=0.1$, and $\tau=1: R / L_{T}=3$ (background) and $R / L_{T}=4$ (strong toroidal branch). The spectra of the two instabilities are shown in figure 18 . We first notice that both the background and toroidal branch modes occur approximately at the same ion scale, $k_{y} \rho_{s} \approx 0.3$. This does not necessarily imply that the two modes would be destabilised for the same $R / L_{T}$. Let us now consider the non-resonant limit. We take the fluid limit of the ITG equation (see (19) of Zocco et al. (2016), or the Fourier transform of (12) of Connor \& Taylor (1987), or (B1) of Candy, Waltz \& Rosenbluth (2004)), neglect 


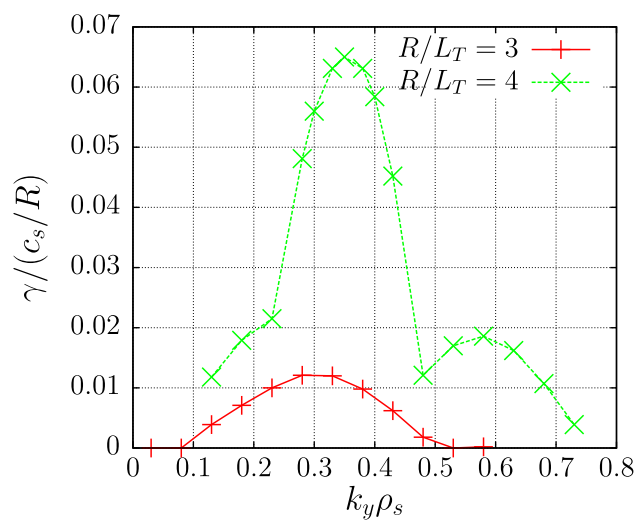

FIGURE 18. Spectra of instability for an $\hat{s}-\alpha$ tokamak, $\epsilon=0.1, \tau=1, R / L_{T}=3$ (background) and $R / L_{T}=4$ (strong toroidal branch).

altogether the shear, i.e. $\hat{s} \equiv 0$, and restore the streaming term, to obtain the Mathieu equation

$$
\frac{\mathrm{d}^{2} \varphi}{\mathrm{d} z^{2}}=[2 q(\omega) \cos 2 z-a(\omega)] \varphi,
$$

with $q(\omega)=8 \omega \omega_{\kappa}^{(0)} /\left(v_{\text {thi }}^{2} / \ell_{\|}^{2}\right), \quad a(\omega)=-8\left(\omega^{3} / \omega_{T} v_{\text {thi }}^{2} / \ell_{\|}^{2}\right)\left[\tau-\omega_{T} k_{y}^{2} \rho_{i}^{2} /(2 \omega)\right]$ and $z=l / 2$, where $l$ is the field-following coordinate and $\ell_{\|}$is the parallel connection length. Basically, when the system approaches marginality, the toroidal driving term (proportional to $q$, not to be confused with the safety factor), becomes smaller and the second-order derivative associated with the streaming term cannot be neglected. The absence of secular terms due to very low shear, however, makes this non-resonant analysis still valid. The resonant theory of such modes does not seem straightforward.

Equation (4.1) has been studied in the context of formation of internal transport barriers in ITG turbulence by Candy et al. (2004) and by Connor \& Hastie (2004). A more general form was investigated by Taylor \& Wilson (1996). In stellarator research, 'weakly localised' Mathieu solutions were first identified by Bhattacharjee et al. (1983).

The most general solution of (4.1) is

$$
\varphi(z)=\mathrm{e}^{\mu z} \Phi(z)
$$

where $\Phi$ is periodic with period $\pi k$, with $k$ integer. The evaluation of $\mu$ was first published in 1886 by Hill (1886), and it will now be applied to the ITG case. One introduces the Fourier series for the $\pi$-period solution

$$
\varphi_{\pi}(z)=\mathrm{e}^{\mu z} \sum_{n=-\infty}^{\infty} b_{2 n} \mathrm{e}^{2 n \mathrm{i} z},
$$

to obtain

$$
(\mu+2 n \mathrm{i})^{2} b_{2 n}+a b_{2 n}-q\left(b_{2 n-2}+b_{2 n+2}\right)=0 .
$$

For a $2 \pi$-period solution, $\varphi_{2 \pi}(z)=\mathrm{e}^{\mu z} \sum_{n=-\infty}^{\infty} b_{2 n}^{\prime} \exp [\mathrm{i} n z]$, we have

$$
(\mu+n \mathrm{i})^{2} c_{n}^{\prime}+a c_{n}^{\prime}-\left(c_{n-1}^{\prime}+c_{n+1}^{\prime}\right)=0,
$$


where $c_{n}^{\prime} \equiv b_{2 n}^{\prime}$. Equation (4.5) is, for $\mu=0$, see (21) of Connor \& Taylor (1987). It is clear that $\mu=0$ is the eigenvalue condition, because twisting boundary conditions require $\varphi^{\prime}(0)=\mu \Phi(0)+\Phi^{\prime}(0)=\mu \Phi(0)=0$, which, indeed, implies $\mu=0$. Similarly, we introduce $c_{n} \equiv b_{2 n}$ and write (4.4) in matrix form, $A_{n m} c_{n}$, where (for $\pi$-period solutions), after setting $\mu=0$,

$$
A_{m n}=\left(\begin{array}{ccccc}
\ddots & -\frac{q}{a-4 \times 2^{2}} & & & \\
-\frac{q}{a-4 \times 1^{2}} & 1 & -\frac{q}{a-4 \times 1^{2}} & & \\
& -\frac{q}{a-4 \times 0^{2}} & 1 & -\frac{q}{a-4 \times 0^{2}} & \\
& & -\frac{q}{a-4 \times 1^{2}} & 1 & -\frac{q}{a-4 \times 1^{2}} \\
& & & -\frac{q}{a-4 \times 2^{2}} & \ddots
\end{array}\right)
$$

is an infinite tridiagonal matrix. The eigenvalue, $\hat{\omega}=\omega /\left(v_{\text {thi }} / a\right)$, is therefore determined from

$$
\operatorname{det} A_{m n}(\hat{\omega})=0 \text {. }
$$

Notice that for $q \sim a$, the off-diagonal elements decay like $m^{-2}$, so the determinant of the matrix is convergent for $m \rightarrow \infty$. For a given large $M,-M \leqslant(m, n) \leqslant M$, one can use the iterative formula for determinants of tridiagonal matrices, to show that (4.7) can be written in terms of the truncated continued fraction

$$
\begin{aligned}
& 1-\frac{q^{2}}{\left(a-4 M^{2}\right)\left[a-4(M-1)^{2}\right]} \\
& \times \frac{q^{2}}{1-\frac{1}{\left[a-4(M-1)^{2}\right]\left[a-4(M-2)^{2}\right]}} \frac{1}{1-\frac{q^{2}}{\left[a-4(M-2)^{2}\right]\left[a-4(M-3)^{2}\right]} \frac{1}{1} .} \\
& =0 \text {, }
\end{aligned}
$$

where the recursive formula is iterated from $m=M$, to $m=-M$, with $\operatorname{det} A_{-M-1} \equiv 1$. Then

$$
\begin{aligned}
& \lim _{M \rightarrow \infty} \frac{q^{2}}{\left(a-4 M^{2}\right)\left[a-4(M-1)^{2}\right]} \\
& \times \frac{q^{2}}{1-\frac{1}{\left[a-4(M-1)^{2}\right]\left[a-4(M-2)^{2}\right]}} \frac{1}{1-\frac{q^{2}}{\left[a-4(M-2)^{2}\right]\left[a-4(M-3)^{2}\right]} \frac{1}{1-\ddots}}
\end{aligned}
$$$$
=1
$$

is the eigenvalue equation for the Floquet ITG mode. Candy et al. (2004) report an eigenvalue condition which reads

$$
a(\hat{\omega})=-2 q(\hat{\omega})+2 q^{1 / 2}(\hat{\omega})
$$

and is valid only for $q \gg 1$. Connor \& Taylor (1987) derived, for large $q$,

$$
a(\hat{\omega})=-2 q(\hat{\omega})+q^{1 / 2}(\hat{\omega}),
$$




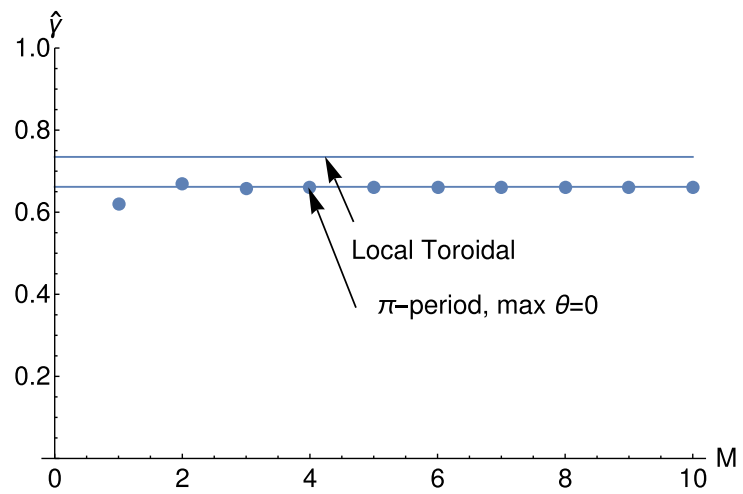

FIGURE 19. Numerical solution of (4.7) and (4.13) derived for $\pi$-periodic solutions with maximum at $\theta \propto z=0$. The eigenvalue of the local toroidal branch is given by the solution of $a=2 q$, where $a$ and $q$ are defined in the text.

after expanding a finite $\hat{s}$ version of (4.1) in Fourier harmonics, and treating the Fourier index as a continuous variable. Following Connor and Taylor, we can consider slowly varying $c_{m}$ coefficients, for the $\pi$-period solution, and obtain

$$
q \frac{\mathrm{d}^{2} c_{m}}{\mathrm{~d} m^{2}}+\left(4 m^{2}-a-2 q\right) c_{m}=0
$$

By using the ansatz $c_{m}=\exp \left[-\lambda m^{2}\right]$, the eigenvalue condition,

$$
a(\hat{\omega})=2 q(\hat{\omega})+2 i q^{1 / 2}(\hat{\omega})
$$

is derived. This corresponds to equation (B9) of Candy et al. (2004) for $q \rightarrow-q$ ((4.10) in this work). In fact, both eigenvalue equations (4.5) and (4.13) are approximate forms of the exact one, for a different periodicity of $2 \pi$ or $\pi$, respectively. For each periodicity, we have two eigenmodes (and eigenvalues) depending on the argument of $q$. For instance, the $2 \pi$-period solutions have maxima at the poloidal location $\theta=\pi$, equation (4.11) and $\theta=0((4.11)$ with $q \rightarrow-q)$.

The numerical solution of $(4.7), \hat{\gamma}=\operatorname{Im}[\hat{\omega}]$, is plotted in figure 19 as a function of the matrix dimension for $\omega_{\kappa}^{(0)} /\left(v_{\text {thi }} / a\right)=0.45, \omega_{T} /\left(v_{\text {thi }} / a\right)=0.6, v_{\text {thi }} / \ell_{\|}=0.5\left(v_{\text {thi }} / a\right)$ and $k_{y} \rho_{i}=0.3$ (here $a$ is a normalising length). The result is compared to the solution of (4.13). In this case $q=10$. The agreement is excellent. For $q \sim 1$, which is the relevant regime, since (4.1) is derived for $\omega_{\kappa} / \omega \sim v_{\mathrm{thi}}^{2} / \ell_{\|}^{2} \ll 1$, equation (4.7) is the correct eigenvalue equation. It is worth mentioning that Taylor \& Hastie (1968), in their seminal work where linear gyrokinetics was derived for the first time for electrostatic perturbations in a torus, report an eigenvalue equation of the form of our (4.7). Their eigenvalue equation (50) reduces to a determinant of a tridiagonal matrix (derived by Coppi et al. 1968), when the plane slab ITG is modified by a sinusoidal periodic gravity term. Coppi et al. recognised the role of the Hill determinant in the evaluation of the true eigenvalue given by the solution of (4.7), but the approximated eigenvalue of (4.11) was derived only 19 years later! We now see that the resonant destabilisation of Floquet modes in stellarators can possibly be compared with the final result derived by Coppi et al. (1968) in appendix B of their work, if temperature-gradient effects are included. 


\section{Discussion and conclusions}

The aim of this work was to elucidate what sets the linear threshold for destabilisation of the electrostatic ion-temperature-gradient driven mode in toroidal magnetic fusion devices. We study both axisymmetric (tokamaks) and non-axisymmetric (stellartors) confinement concepts.

In regimes of interest for the stellarator Wendelstein 7-X, a background of unstable modes exists below the threshold of destabilisation of the toroidal branch of the ITG mode. We proposed the hypothesis that such modes are ITG modes of the Floquet type. The eigenfunctions resulting from GENE numerical simulations of Wendelstein 7-X show a much slower decay along the field line for these modes than for modes of the toroidal branch (see figure 14). This behaviour is evocative of the prediction of weakly localised drift-wave modes in stellarators, described in terms of Mathieu functions by Bhattacharjee et al. (1983). In the non-resonant limit, a complete eigenvalue equation is then proposed and studied in relation to previously known results.

The importance of our findings relies on the fact that such modes result from having a very low global shear, $\hat{s}$, and feature similar qualitative behaviour in tokamaks and stellarators. In the former case, one could imagine them being relevant for advanced confinement scenarios with very flat safety factor profiles. For finite density gradients, the insensitivity of the derived temperature gradient thresholds to the temperature ratio $\tau=T_{i} / T_{e}$ for $\tau<1$ seems a rather clear effect, distinguishing these modes from the more conventional branches. For stellarators, our findings could have a non-negligible impact on confinement if Floquet solutions play any role nonlinearly. If stellarator electrostatic turbulence is affected by the nonlinear activity of these modes, there is an open question regarding what is really the achievable temperature gradient for a stellarator with low global shear, as opposed to known tokamak cases, where a finite turbulent transport is observed above a finite critical temperature gradient, generally larger than the one discussed in this work (Dimits et al. 2000). Additional stabilising effects can of course suppress these modes. Our results are perhaps also relevant for trapped-electron mode driven turbulence, where similar eigenfunctions and 'background' modes can be observed (Proll, Xanthopoulos \& Helander 2013; Faber et al. 2015).

\section{Acknowledgements}

Part of this work was presented at the first conference 'Frontiers in Plasma Physics', held at the Abbey of the Most Holy Trinity of Spineto (Sarteano, Italy) under the auspices of the Journal of Plasma Physics (Cambridge University Press).

\section{Appendix A. Local kinetic limit formulation}

In order to solve the integral $I_{i}$ for arbitrary ion Larmor radii, some preliminary manipulations are in order. We add and subtract $\omega_{\kappa} \hat{v}_{\|}^{2}+\omega_{B} \hat{v}_{\perp}^{2} / 2$ in the numerator of $I_{i}$ to obtain

$$
\begin{aligned}
I_{i}= & 2 \int_{0}^{\infty} \mathrm{d} \hat{v}_{\perp} \hat{v}_{\perp} J_{0}^{2}\left(\boldsymbol{k}_{\perp}^{2} \rho_{i}^{2} \hat{v}_{\perp}^{2}\right) \mathrm{e}^{-\hat{v}_{\perp}^{2}}-\frac{\omega_{* i}}{\omega}\left(1-\frac{3}{2} \eta_{i}\right) J^{(0)} \\
& +\frac{\omega_{\kappa}-\omega_{* i} \eta_{i}}{\omega} J_{\perp}^{(2)}+\frac{\omega_{B} / 2-\omega_{* i} \eta_{i}}{\omega} J_{\|}^{(2)}
\end{aligned}
$$


with

$$
J_{\perp, \|}^{(n)}=2 \int_{0}^{\infty} \mathrm{d} \hat{v}_{\perp} \hat{v}_{\perp} \int_{-\infty}^{\infty} \mathrm{d} \hat{v}_{\|} \hat{v}_{\perp, \|}^{n} \frac{J_{0}^{2}\left(\boldsymbol{k}_{\perp}^{2} \rho_{i}^{2} \hat{v}_{\perp}^{2}\right)}{1-\left(\frac{\omega_{\kappa}}{\omega} \hat{v}_{\|}^{2}+\frac{\omega_{B}}{\omega} \hat{v}_{\perp}^{2} / 2\right)} \frac{\mathrm{e}^{-\left(\hat{v}_{\|}^{2}+\hat{v}_{\perp}^{2}\right)}}{\sqrt{\pi}} .
$$

The first line of (A 1) gives the well-known result of gyrokinetic theory:

$$
2 \int_{0}^{\infty} \mathrm{d} \hat{v}_{\perp} \hat{v}_{\perp} J_{0}^{2}\left(\boldsymbol{k}_{\perp}^{2} \rho_{i}^{2} \hat{v}_{\perp}^{2}\right) \mathrm{e}^{-\hat{v}_{\perp}^{2}}=\Gamma_{0}(b),
$$

where $b=\boldsymbol{k}_{\perp}^{2} \rho_{i}^{2} / 2$, and $\Gamma_{0}(b)=\mathrm{I}_{0}(b) \exp (-b)$, with $\mathrm{I}_{0}$ the modified Bessel function. We now integrate the resonances in a way similar to that presented by Biglari, Diamond and Rosenbluth (BDR) (Biglari et al. 1989). For $J^{(0)}$ we have

$$
\begin{aligned}
J^{(0)} & =-2 \mathrm{i} \int_{0}^{\infty} \mathrm{d} \hat{v}_{\perp} \hat{v}_{\perp} J_{0}^{2} \int_{-\infty}^{\infty} \frac{\mathrm{d} \hat{v}_{\|}}{\sqrt{\pi}} \int_{0}^{\infty} \mathrm{d} \lambda \exp \left(\mathrm{i} \lambda\left[1-\left(\frac{\omega_{\kappa}}{\omega} \hat{v}_{\|}^{2}+\frac{\omega_{B}}{\omega} \hat{v}_{\perp}^{2} / 2\right)\right]-\left(\hat{v}_{\|}^{2}+\hat{v}_{\perp}^{2}\right)\right) \\
& =-2 \mathrm{i} \int_{0}^{\infty} \mathrm{d} \lambda \mathrm{e}^{\mathrm{i} \lambda} \int_{0}^{\infty} \mathrm{d} \hat{v}_{\perp} \hat{v}_{\perp} J_{0}^{2} \int_{-\infty}^{\infty} \frac{\mathrm{d} \hat{v}_{\|}}{\sqrt{\pi}} \mathrm{e}^{-\hat{v}_{\perp}^{2}\left(1+\mathrm{i} \lambda\left(\omega_{B} / 2 \omega\right)\right)} \mathrm{e}^{-\hat{v}_{\|}^{2}\left(1+\mathrm{i} \lambda\left(\omega_{\kappa} / \omega\right)\right)} \\
& =-2 \mathrm{i} \int_{0}^{\infty} \mathrm{d} \lambda \mathrm{e}^{\mathrm{i} \lambda} \int_{0}^{\infty} \mathrm{d} \hat{v}_{\perp} \hat{v}_{\perp} J_{0}^{2} \frac{\mathrm{e}^{-\hat{v}_{\perp}^{2}\left(1+\mathrm{i} \lambda\left(\omega_{B} / 2 \omega\right)\right)}}{\left(1+\mathrm{i} \lambda \frac{\omega_{\kappa}}{\omega}\right)^{1 / 2}} \\
& =-\mathrm{i} \int_{0}^{\infty} \mathrm{d} \lambda \frac{\mathrm{e}^{\mathrm{i} \lambda}}{\left(1+\mathrm{i} \lambda \frac{\omega_{\kappa}}{\omega}\right)^{1 / 2}} \frac{\Gamma_{0}(\hat{b})}{1+\mathrm{i} \lambda \frac{\omega_{B}}{2 \omega}},
\end{aligned}
$$

with $\hat{b}=b /\left[1+\mathrm{i} \lambda \omega_{B} /(2 \omega)\right]$. We require

$$
\begin{aligned}
& \operatorname{Im}\left[\lambda \frac{\omega_{\kappa}}{\omega}\right]<1, \\
& \operatorname{Im}\left[\lambda \frac{\omega_{B}}{2 \omega}\right]<1,
\end{aligned}
$$

and

$$
\operatorname{Im}[\lambda]>0
$$

in order to guarantee convergence of the velocity space and $\lambda$ integrals, respectively. Conditions (A 5)-(A 7) thus define an abscissa of convergence in the complex $\lambda$-plane

$$
\operatorname{Re}[\lambda]>\alpha_{c} \equiv \operatorname{Im}[\omega]^{-1}\left(\operatorname{Im}[\lambda] \operatorname{Re}[\omega]-|\omega|^{2} / \omega_{\kappa}\right) .
$$

Here we are using $\omega_{B} \equiv \omega_{\kappa}$ (valid in the low- $\beta$ case, where $\beta$ is the ratio of kinetic and magnetic plasma pressure) and taking the most restrictive of conditions (A 5)(A 7). Similarly, we obtain

$$
J_{\|}^{(2)}=-\frac{\mathrm{i}}{2} \int_{0}^{\infty} \mathrm{d} \lambda \frac{\mathrm{e}^{\mathrm{i} \lambda}}{\left(1+\mathrm{i} \lambda \frac{\omega_{\kappa}}{\omega}\right)^{3 / 2}} \frac{\Gamma_{0}(\hat{b})}{1+\mathrm{i} \lambda \frac{\omega_{B}}{2 \omega}},
$$

and

$$
J_{\perp}^{(2)}=-\mathrm{i} \int_{0}^{\infty} \mathrm{d} \lambda \frac{\mathrm{e}^{\mathrm{i} \lambda}}{\left(1+\mathrm{i} \lambda \frac{\omega_{\kappa}}{\omega}\right)^{1 / 2}} \frac{\Gamma_{0}(\hat{b})+\hat{b}\left[\Gamma_{1}(\hat{b})-\Gamma_{0}(\hat{b})\right]}{\left(1+\mathrm{i} \lambda \frac{\omega_{B}}{2 \omega}\right)^{2}} .
$$


We now introduce the Padé approximants

$$
\Gamma_{0}(\hat{b}) \approx \frac{1}{1+\frac{b}{1+\mathrm{i} \lambda \frac{\omega_{B}}{2 \omega}}},
$$

and

$$
\Gamma_{0}(\hat{b})+\hat{b}\left[\Gamma_{1}(\hat{b})-\Gamma_{0}(\hat{b})\right] \approx \frac{1}{\left(1+\frac{b}{1+\mathrm{i} \lambda \frac{\omega_{B}}{2 \omega}}\right)^{2}}
$$

which will allow us to perform the $\lambda$ integration. The integral $J^{(0)}$ becomes

$$
J^{(0)}=-\mathrm{i} \int_{0}^{\infty} \mathrm{d} \lambda \frac{\mathrm{e}^{\mathrm{i} \lambda}}{\left(1+\mathrm{i} \lambda \frac{\omega_{\kappa}}{\omega}\right)^{1 / 2}} \frac{1}{1+\mathrm{i} \lambda \frac{\omega_{B}}{2 \omega}+b} .
$$

For $\lambda \rightarrow \lambda \omega / \omega_{\kappa}, b \rightarrow 0$, and $\omega_{B} \equiv \omega_{\kappa}$,

$$
\begin{aligned}
\lim _{b \rightarrow 0} J^{(0)} & =-\mathrm{i} \frac{\omega}{\omega_{\kappa}} \int_{0}^{\infty} \mathrm{d} \lambda \frac{\mathrm{e}^{\mathrm{i}\left(\omega / \omega_{\kappa}\right) \lambda}}{(1+\mathrm{i} \lambda)^{1 / 2}} \frac{1}{1+\mathrm{i} \frac{\lambda}{2}} \\
& =\frac{\omega}{\omega_{\kappa}} F_{1,1},
\end{aligned}
$$

with $F_{1,1}$ defined in (A2) of BDR. We notice that the change of variables $\lambda \rightarrow \lambda \omega / \omega_{\kappa}$ requires $(\operatorname{Re}[\lambda] \operatorname{Im}[\omega]+\operatorname{Im}[\lambda] \operatorname{Re}[\omega]) / \omega_{\kappa}>0$ for the convergence of the $\lambda$ integral. At marginal stability, for $\omega_{\kappa}<0$ and $\omega<0$, this is condition (A 7).

\section{Appendix B. Arbitrary Larmor radii solution}

For $b \neq 0$, the analytical technique used by BDR to solve for $F_{1,1}$ cannot be used to perform the integration. We prefer to proceed in a different way.

Let us consider

$$
R=-\mathrm{i} \int_{0}^{\infty} \mathrm{d} \lambda \frac{\mathrm{e}^{\mathrm{i} \Omega \lambda}}{(1+\mathrm{i} \lambda)^{1 / 2}} \frac{1}{1+\mathrm{i} \lambda \frac{\omega_{B}}{2 \omega_{\kappa}}+b},
$$

with $\Omega=\omega / \omega_{\kappa}$. We change variables

$$
\mathrm{i} \lambda=t^{2}-1,
$$

and obtain

$$
\begin{aligned}
R & =-4 \frac{\omega_{\kappa}}{\omega_{B}} \int_{1}^{\mathrm{e}^{\mathrm{i}(\pi / 4)} \infty} \mathrm{d} t \frac{\mathrm{e}^{\Omega\left(t^{2}-1\right)}}{2 \frac{\omega_{\kappa}}{\omega_{B}}(1+b)-1+t^{2}} \\
& =-4 \frac{\omega_{\kappa}}{\omega_{B}} \mathrm{e}^{-\Omega} \int_{1 / \sqrt{g}}^{\mathrm{e}^{\mathrm{i}(\pi / 4)} \infty} \frac{\mathrm{d} u}{\sqrt{g}} \frac{\mathrm{e}^{\Omega g u^{2}}}{1+u^{2}},
\end{aligned}
$$


with

$$
g=2 \frac{\omega_{\kappa}}{\omega_{B}}(1+b)-1 .
$$

In the case $\omega_{\kappa}=\omega_{B}, g=1+2 b>0 \forall b$. We split the domain of integration and define

$$
\begin{aligned}
R & =-4 \frac{\omega_{\kappa}}{\omega_{B}} \mathrm{e}^{-\Omega}\left(\int_{0}^{\mathrm{i}} \mathrm{e}^{\mathrm{i}(\pi / 4) \infty} \frac{\mathrm{d} u}{\sqrt{g}}-\int_{0}^{1 / \sqrt{g}} \frac{\mathrm{d} u}{\sqrt{g}}\right) \frac{\mathrm{e}^{\Omega g u^{2}}}{1+u^{2}} \\
& \equiv-4 \frac{\omega_{\kappa}}{\omega_{B}} \mathrm{e}^{-\Omega}\left(I_{\infty}-I_{g}\right) .
\end{aligned}
$$

In appendices $\mathrm{C}$ and $\mathrm{D}$, we show that

$$
R=-4 \frac{\omega_{\kappa}}{\omega_{B}} \frac{\mathrm{e}^{-\Omega}}{\sqrt{g}}\left\{-\frac{\mathrm{i}}{2} \sqrt{\pi} Z(\sqrt{g \Omega})-2 \pi \mathrm{e}^{-g \Omega} T\left[\mathrm{i} \sqrt{2 g \Omega} ; \frac{1}{\sqrt{g}}\right]\right\},
$$

where $Z$ is the plasma dispersion function (Fried \& Conte 1961), and $T$ the Owen $T$-function (Owen 1956). By direct comparison of (A 13) and (B 1), we have

$$
J^{(0)}=\frac{\omega}{\omega_{\kappa}} R
$$

The integral $J_{\perp}^{(2)}$ now becomes

$$
\begin{aligned}
J_{\perp}^{(2)} & =-\mathrm{i} \int_{0}^{\infty} \mathrm{d} \lambda \frac{\mathrm{e}^{\mathrm{i} \lambda}}{\left(1+\mathrm{i} \lambda \frac{\omega_{\kappa}}{\omega}\right)^{1 / 2}} \frac{1}{\left(1+\mathrm{i} \lambda \frac{\omega_{B}}{2 \omega}+b\right)^{2}} \\
& =-\frac{\mathrm{d}}{\mathrm{d} b} J^{(0)} .
\end{aligned}
$$

To evaluate the integral $J_{\|}^{(2)}$, it is convenient to consider a simple generalisation of the auxiliary integral, $R$, namely

$$
R_{\nu}=-\mathrm{i} \int_{0}^{\infty} \mathrm{d} \lambda \frac{\mathrm{e}^{\mathrm{i} \Omega \lambda}}{(\nu+\mathrm{i} \lambda)^{1 / 2}} \frac{1}{1+\mathrm{i} \lambda \frac{\omega_{B}}{2 \omega_{\kappa}}+b} .
$$

Then

$$
J_{\|}^{(2)}=-\lim _{\nu \rightarrow 1} \Omega \frac{\mathrm{d}}{\mathrm{d} \nu} R_{\nu} .
$$

The integral $R_{v}$ can be related to $R$ after some straightforward algebra. We find

$$
\begin{aligned}
R_{\nu} & =-4 \frac{\omega_{\kappa}}{\omega_{B}} \mathrm{e}^{-\nu \Omega} \int_{\sqrt{\nu / g_{\nu}}}^{\mathrm{e}^{\mathrm{i}(\pi / 4) \infty}} \frac{\mathrm{d} u}{\sqrt{g_{\nu}}} \frac{\mathrm{e}^{g_{\nu} \Omega u^{2}}}{1+u^{2}} \\
& =-4 \frac{\omega_{\kappa}}{\omega_{B}} \frac{\mathrm{e}^{-v \Omega}}{\sqrt{g_{v}}}\left\{-\frac{\mathrm{i}}{2} \sqrt{\pi} Z\left(\sqrt{g_{\nu} \Omega}\right)-2 \pi \mathrm{e}^{-g_{\nu} \Omega} T\left[\mathrm{i} \sqrt{2 g_{\nu} \Omega} ; \sqrt{\frac{\nu}{g_{\nu}}}\right]\right\},
\end{aligned}
$$

with

$$
g_{v}=\frac{2 \omega_{\kappa}}{\omega_{B}}(1+b)-v
$$


Summarising, the local kinetic dispersion relation is

$$
\begin{aligned}
1+\tau= & \Gamma_{0}(b)-\frac{\omega_{* i}}{\omega}\left(1-\frac{3}{2} \eta_{i}\right) J_{\perp}^{(0)} \\
& +\frac{\omega_{* i}}{\omega}\left(\frac{\omega_{\kappa}}{\omega_{* i}}-\eta_{i}\right) J_{\perp}^{(2)}+\frac{\omega_{* i}}{\omega}\left(\frac{\omega_{B}}{2 \omega_{* i}}-\eta_{i}\right) J_{\|}^{(2)},
\end{aligned}
$$

with

$$
\begin{array}{r}
J^{(0)}=\lim _{\nu \rightarrow 1} \frac{\omega}{\omega_{\kappa}} R_{\nu}, \\
J_{\perp}^{(2)}=-\frac{\mathrm{d}}{\mathrm{d} b} J^{(0)}, \\
J_{\|}^{(2)}=-\lim _{\nu \rightarrow 1} \Omega \frac{\mathrm{d}}{\mathrm{d} \nu} R_{\nu},
\end{array}
$$

with $R_{v}$ defined in (B 11), $g_{v}=2(1+b) \omega_{\kappa} / \omega_{B}-v$, and

$$
T\left[\mathrm{i} \sqrt{2 g_{v} \frac{\omega}{\omega_{\kappa}}} ; \sqrt{\frac{\nu}{g_{\nu}}}\right]=\frac{\mathrm{e}^{g_{\nu}\left(\omega / \omega_{\kappa}\right)}}{2 \pi \sqrt{g_{v}}} \sum_{n=0}^{\infty} \frac{\left(-v / g_{v}\right)^{n}}{(2 n+1)} \sum_{m=0}^{n} \frac{\left(-g_{v} \frac{\omega}{\omega_{\kappa}}\right)^{m}}{m !} .
$$

This expression for the Owen $T$-function is derived in appendix D.

\section{Appendix C. The integral $I_{\infty}$}

For $I_{\infty}$, we change variables to

$$
g \Omega u^{2} \rightarrow-\xi^{2},
$$

so that, when $u \rightarrow \mathrm{e}^{\mathrm{i}(\pi / 4)} \infty, \xi \rightarrow \mathrm{e}^{\mathrm{i}((3 / 4) \pi+(\operatorname{argg} \Omega) / 2)} \infty$. A closed form of the integral can be found for an unstable mode $\arg (\Omega g)=\arg \left\{\omega\left[2 \omega_{\kappa}(1+b)-\omega_{B}\right] /\left(\omega_{\kappa} \omega_{B}\right)\right\} \approx \pi / 2$, for $\operatorname{Re}[\omega] \rightarrow 0$. Then, $\xi \rightarrow \mathrm{e}^{\mathrm{i}((3 / 4) \pi+(\operatorname{argg} \Omega) / 2)} \infty \approx \mathrm{e}^{\mathrm{i}((3 / 4) \pi+(\pi / 4))} \infty=-\infty$, and $I_{\infty}$ becomes

$$
\begin{aligned}
I_{\infty} & =-\mathrm{i} \sqrt{\Omega} \int_{0}^{-\infty} \mathrm{d} \xi \frac{\mathrm{e}^{-\xi^{2}}}{(\sqrt{g \Omega})^{2}-\xi^{2}} \\
& =\mathrm{i} \frac{\sqrt{\Omega}}{2} \int_{-\infty}^{\infty} \mathrm{d} \xi \frac{\mathrm{e}^{-\xi^{2}}}{2 \sqrt{g \Omega}}\left\{\frac{1}{\xi+\sqrt{\Omega}}-\frac{1}{\xi-\sqrt{\Omega}}\right\} \\
& =-\frac{\mathrm{i}}{2} \sqrt{\frac{\pi}{g}} Z(\sqrt{g \Omega}) .
\end{aligned}
$$

Analytic continuation is needed for damped modes.

\section{Appendix D. The integral $I_{g}$ and the Owen $T$-function}

In the case of $I_{g}$, we have

$$
I_{g}=\int_{0}^{1 / \sqrt{g}} \frac{\mathrm{d} u}{\sqrt{g}} \frac{\mathrm{e}^{\Omega g u^{2}}}{1+u^{2}} .
$$


This integral can be written in terms of the Owen $T$-function (Owen 1956).

$$
\begin{aligned}
I_{g} & =\int_{0}^{1 / \sqrt{g}} \frac{\mathrm{d} u}{\sqrt{g}} \frac{\mathrm{e}^{\Omega g u^{2}}}{1+u^{2}} \\
& =\mathrm{e}^{-g \Omega} \int_{0}^{1 / \sqrt{g}} \frac{\mathrm{d} u}{\sqrt{g}} \frac{\exp \left(-(\mathrm{i} \sqrt{2 g \Omega})^{2}\left(\frac{u^{2}}{2}+\frac{1}{2}\right)\right)}{1+u^{2}} \\
& =2 \pi \frac{\mathrm{e}^{-g \Omega}}{\sqrt{g}} T\left[\mathrm{i} \sqrt{2 g \Omega} ; \frac{1}{\sqrt{g}}\right] .
\end{aligned}
$$

For analytical manipulations and numerical implementation, the most convenient way of writing the Owen function is, perhaps, the following. Since

$$
T\left[\mathrm{i} \sqrt{2 g_{v} \frac{\omega}{\omega_{\kappa}}} ; \sqrt{\frac{\nu}{g}}\right]=\frac{1}{2 \pi} \int_{0}^{\sqrt{v / g_{v}}} \mathrm{~d} t \frac{\mathrm{e}^{g_{\nu}\left(\omega / \omega_{k}\right)\left(1+t^{2}\right)}}{1+t^{2}},
$$

if we rewrite

$$
\begin{aligned}
T\left[\mathrm{i} \sqrt{2 g_{v}\left(\omega / \omega_{\kappa}\right)} ; \sqrt{\left.\frac{\nu}{g}\right]=}\right. & \frac{1}{2 \pi} \int_{0}^{\sqrt{v / g_{v}}} \mathrm{~d} t \frac{\mathrm{e}^{g_{v}\left(\omega / \omega_{\kappa}\right)\left(1+t^{2}\right)}}{1+t^{2}} \\
& =\frac{1}{2 \pi} \int_{0}^{\infty} \mathrm{d} \xi \int_{0}^{\sqrt{v / g_{v}}} \mathrm{~d} t \mathrm{e}^{-\xi\left(1+t^{2}\right)} \mathrm{e}^{g \nu\left(\omega / \omega_{\kappa}\right)\left(1+t^{2}\right)} \\
& =\frac{1}{2 \pi} \mathrm{e}^{g_{v}\left(\omega / \omega_{k}\right)} \int_{0}^{\infty} \mathrm{d} \xi \mathrm{e}^{-\xi} \int_{0}^{\sqrt{v / g_{v}}} \mathrm{~d} t \mathrm{e}^{-\left(\xi-g\left(\omega / \omega_{k}\right)\right) t^{2}} \\
& =\frac{1}{2 \pi} \mathrm{e}^{g_{v}\left(\omega / \omega_{k}\right)} \int_{0}^{\infty} \mathrm{d} \xi \mathrm{e}^{-\xi} \sqrt{\frac{\pi / 4}{\xi-g_{v} \frac{\omega}{\omega_{\kappa}}}} \operatorname{Erf} \sqrt{v\left(\frac{\xi}{g_{v}}-\frac{\omega}{\omega_{\kappa}}\right)},
\end{aligned}
$$

we are then able to introduce the series representation of the error function, to obtain

$$
\begin{aligned}
T\left[\mathrm{i} \sqrt{2 g_{v}\left(\omega / \omega_{\kappa}\right)} ; \sqrt{\frac{\nu}{g}}\right] & =\frac{\mathrm{e}^{g_{v}\left(\omega / \omega_{k}\right)}}{2 \pi \sqrt{g_{v}}} \int_{0}^{\infty} \mathrm{d} \xi \mathrm{e}^{-\xi} \sum_{n=0}^{\infty} \frac{\left(-v / g_{v}\right)^{n}}{n !(2 n+1)}\left(\xi-g_{v} \frac{\omega}{\omega_{\kappa}}\right)^{n} \\
& =\frac{\mathrm{e}^{g_{v}\left(\omega / \omega_{k}\right)}}{2 \pi \sqrt{g_{v}}} \sum_{n=0}^{\infty} \frac{\left(-v / g_{v}\right)^{n}}{n !(2 n+1)} \int_{0}^{\infty} \mathrm{d} \xi \mathrm{e}^{-\xi}\left(\xi-g_{v} \frac{\omega}{\omega_{\kappa}}\right)^{n} \\
& =\frac{1}{2 \pi \sqrt{g_{v}}} \sum_{n=0}^{\infty} \frac{\left(-v / g_{v}\right)^{n}}{n !(2 n+1)} \int_{-g_{v}\left(\omega / \omega_{k}\right)}^{\infty} \mathrm{d} y \mathrm{e}^{-y} y^{n} \\
& =\frac{1}{2 \pi \sqrt{g_{v}}} \sum_{n=0}^{\infty} \frac{\left(-v / g_{v}\right)^{n}}{n !(2 n+1)}\left(\int_{0}^{\infty} \mathrm{d} y-\int_{0}^{-g_{v}\left(\omega / \omega_{k}\right)} \mathrm{d} y\right) \mathrm{e}^{-y} y^{n} \\
& =\frac{1}{2 \pi \sqrt{g_{v}}} \sum_{n=0}^{\infty} \frac{\left(-v / g_{v}\right)^{n}}{n !(2 n+1)}\left[\Gamma(n+1)-\gamma\left(n+1,-g_{v} \frac{\omega}{\omega_{\kappa}}\right)\right],
\end{aligned}
$$


where

$$
\gamma\left(n+1,-g_{v} \frac{\omega}{\omega_{\kappa}}\right)
$$

is the incomplete gamma function (Tricomi 1950a,b). Tricomi shows that, for integer $n$,

$$
\gamma\left(n+1,-g_{\nu} \frac{\omega}{\omega_{\kappa}}\right)=n !\left[1-\mathrm{e}^{g_{\nu}\left(\omega / \omega_{\kappa}\right)} \sum_{m=0}^{n} \frac{\left(-g_{\nu} \frac{\omega}{\omega_{\kappa}}\right)^{m}}{m !}\right],
$$

therefore, our series representation of the Owen function is

$$
T\left[\mathrm{i} \sqrt{2 g_{v} \frac{\omega}{\omega_{\kappa}}} ; \sqrt{\frac{v}{g_{\nu}}}\right]=\frac{\mathrm{e}^{g_{\nu}\left(\omega / \omega_{\kappa}\right)}}{2 \pi \sqrt{g_{v}}} \sum_{n=0}^{\infty} \frac{\left(-v / g_{\nu}\right)^{n}}{(2 n+1)} \sum_{m=0}^{n} \frac{\left(-g_{v}\left(\omega / \omega_{\kappa}\right)\right)^{m}}{m !} .
$$

\section{Appendix E. Proof of some identities}

In appendix $\mathrm{B}$, we derived the following result

$$
R=-4 \frac{\omega_{\kappa}}{\omega_{B}} \frac{\mathrm{e}^{-\Omega}}{\sqrt{g}}\left\{-\frac{\mathrm{i}}{2} \sqrt{\pi} Z(\sqrt{g \Omega})-2 \pi \mathrm{e}^{-g \Omega} T\left[\mathrm{i} \sqrt{2 g \Omega} ; \frac{1}{\sqrt{g}}\right]\right\},
$$

which is valid $\forall b$. We now prove that, for $\omega_{\kappa} \equiv \omega_{B}$,

$$
\lim _{b \rightarrow 0} R=F_{1,1}
$$

where

$$
F_{1,1}^{1 / 2}=\mathrm{e}^{-\Omega} \int_{-\infty}^{\Omega} \mathrm{d} z \frac{\mathrm{e}^{-z}}{z^{1 / 2}}
$$

is the result found by BDR (Biglari et al. 1989).

Since $\lim _{b \rightarrow 0} g=1$, we have

$$
\lim _{b \rightarrow 0} R=2 \mathrm{i} \sqrt{\pi} \mathrm{e}^{-\Omega} Z(\sqrt{\Omega})+4 \pi \mathrm{e}^{-2 \Omega} \Phi(\mathrm{i} \sqrt{2 \Omega})[1-\Phi(\mathrm{i} \sqrt{2 \Omega})],
$$

where

$$
\Phi(x)=\frac{1}{\sqrt{2 \pi}} \int_{-\infty}^{x} \mathrm{~d} t \mathrm{e}^{-t^{2} / 2}
$$

It is easy to see that

$$
\begin{aligned}
\Phi(x) & =\frac{\mathrm{i}}{2 \sqrt{\pi}} \int_{-\infty}^{\Omega} \mathrm{d} z \frac{\mathrm{e}^{-z}}{z^{1 / 2}} \\
& =\frac{\mathrm{i}}{2 \sqrt{\pi}} \mathrm{e}^{\Omega} F_{1,1}^{1 / 2} .
\end{aligned}
$$


Then

$$
\begin{aligned}
\lim _{b \rightarrow 0} R & =2 \mathrm{i} \sqrt{\pi} \mathrm{e}^{-\Omega} Z(\sqrt{\Omega})+4 \pi \mathrm{e}^{-2 \Omega} \Phi(\mathrm{i} \sqrt{2 \Omega})[1-\Phi(\mathrm{i} \sqrt{2 \Omega})], \\
& =2 \mathrm{i} \sqrt{\pi} \mathrm{e}^{-\Omega}\left\{Z(\sqrt{\Omega})+F_{1,1}^{1 / 2}\right\}+F_{1,1} .
\end{aligned}
$$

However, it is also true that

$$
\begin{aligned}
F_{1,1}^{1 / 2} & =\mathrm{e}^{-\Omega} \int_{-\infty}^{\Omega} \mathrm{d} z \frac{\mathrm{e}^{-z}}{z^{1 / 2}} \\
& =-2 \mathrm{ie}^{-\Omega} \int_{-\infty}^{\mathrm{i} \Omega^{1 / 2}} \mathrm{~d} t \mathrm{e}^{-t^{2}} \\
& =-Z(\sqrt{\Omega}),
\end{aligned}
$$

then the first two terms in equation (E7) cancel, leaving us with

$$
\lim _{b \rightarrow 0} R=F_{1,1}
$$

which is what we aimed to prove.

\section{Appendix F. Real frequency at marginality, long-wavelength limit $b \rightarrow 0$}

In the light of the identities just proved in appendix E, we are in the position to say that, for $b \rightarrow 0$,

$$
\begin{aligned}
D \equiv & -(1+\tau)+I_{i} \\
= & -(1+\tau)+\left(1-\frac{\omega_{* i}}{\omega}\right) \Omega Z^{2}(\sqrt{\Omega}) \\
& +\eta_{i} \frac{\omega_{* i}}{\omega}\left\{(1-2 \Omega) \Omega Z^{2}(\sqrt{\Omega})-2 \Omega^{3 / 2} Z(\sqrt{\Omega})\right\},
\end{aligned}
$$

which corresponds to $D_{0}$ in (3) of BDR (Biglari et al. 1989). The real frequency at marginality is evaluated by solving $\operatorname{Im}\left[D\left(\omega_{r}\right)\right] \equiv D_{i}\left(\omega_{r}\right)=0$, where we set $\omega=\omega_{r}+\mathrm{i} \gamma$, and take $\gamma \rightarrow 0$. This comes directly from (F1)

$$
\left\{1-\frac{\omega_{* i}}{\omega_{r}}+\eta_{i} \frac{\omega_{* i}}{\omega_{r}}\left(1-2 \frac{\omega_{r}}{\omega_{\kappa}}\right)\right\} \operatorname{Re}\left[Z\left(\sqrt{\frac{\omega_{r}}{\omega_{\kappa}}}\right)\right]=\eta_{i} \frac{\omega_{* i}}{\sqrt{\omega_{r} \omega_{\kappa}}} .
$$

As a validity check, one can set $J_{0}^{2} \rightarrow 1$ in the definition of $I_{i}$ and integrate the resonances in yet another way. Thus

$$
\begin{aligned}
I_{i}= & 2 \int_{0}^{\infty} \mathrm{d} \hat{v}_{\perp} \hat{v}_{\perp} \mathrm{e}^{-\hat{v}_{\perp}^{2}} \int_{-\infty}^{\infty} \frac{\mathrm{d} \hat{v}_{\|} \mathrm{e}^{-\hat{v}_{\perp}^{2}}}{\sqrt{\pi}} \frac{\Omega-\Omega_{*}\left[1+\eta_{i}\left(\hat{v}_{\|}^{2}+\hat{v}_{\perp}^{2}-3 / 2\right)\right]}{\Omega-\left(\hat{v}_{\|}^{2}+\hat{v}_{\perp}^{2}\right)} \\
= & -2 \int_{0}^{\infty} \mathrm{d} \hat{v}_{\perp} \hat{v}_{\perp} \mathrm{e}^{-\hat{v}_{\perp}^{2}}\left\{\Omega-\Omega_{* i}\left(1-\frac{3}{2} \eta_{i}\right) \frac{Z\left(\sqrt{\Omega-\hat{v}_{\perp}^{2} / 2}\right)}{\sqrt{\Omega-\hat{v}_{\perp}^{2} / 2}}\right\} \\
& +\eta_{i} \Omega_{* i}\left\{1+2 \int_{0}^{\infty} \mathrm{d} \hat{v}_{\perp} \hat{v}_{\perp}^{3} \mathrm{e}^{-\hat{v}_{\perp}^{2}} \frac{Z\left(\sqrt{\Omega-\hat{v}_{\perp}^{2} / 2}\right)}{\sqrt{\Omega-\hat{v}_{\perp}^{2} / 2}}\right\} \\
& +\eta_{i} \Omega_{* i} 2 \int_{0}^{\infty} \mathrm{d} \hat{v}_{\perp} \hat{v}_{\perp} \mathrm{e}^{-\hat{v}_{\perp}^{2}} \sqrt{\Omega-\hat{v}_{\perp}^{2} / 2 Z}\left(\sqrt{\Omega-\hat{v}_{\perp}^{2} / 2}\right) .
\end{aligned}
$$


It is easy to see that

$$
\begin{aligned}
& 2 \int_{0}^{\infty} \mathrm{d} \hat{v}_{\perp} \hat{v}_{\perp} \mathrm{e}^{-\hat{v}_{\perp}^{2}} \frac{Z\left(\sqrt{\Omega-\hat{v}_{\perp}^{2} / 2}\right)}{\sqrt{\Omega-\hat{v}_{\perp}^{2} / 2}}=-4 \mathrm{ie}^{-2 \Omega} \int_{\mathrm{i} \Omega^{1 / 2}}^{\infty} \mathrm{d} \zeta \mathrm{e}^{-2 \zeta^{2}} Z(\mathrm{i} \zeta) \equiv I_{1}, \\
& 2 \int_{0}^{\infty} \mathrm{d} \hat{v}_{\perp} \hat{v}_{\perp}^{3} \mathrm{e}^{-\hat{v}_{\perp}^{2}} \frac{Z\left(\sqrt{\Omega-\hat{v}_{\perp}^{2} / 2}\right)}{\sqrt{\Omega-\hat{v}_{\perp}^{2} / 2}}=2 \int_{0}^{\infty} \mathrm{d} \hat{v}_{\perp} 2 \hat{v}_{\perp}\left(\frac{\hat{v}_{\perp}^{2}}{2}-\Omega+\Omega\right) \\
& \times \mathrm{e}^{-\hat{v}_{\perp}^{2}} \frac{Z\left(\sqrt{\Omega-\hat{v}_{\perp}^{2} / 2}\right)}{\sqrt{\Omega-\hat{v}_{\perp}^{2} / 2}} \\
& =2 \Omega I_{1}+2(-4 \mathrm{i}) \mathrm{e}^{-2 \Omega} \int_{\mathrm{i} \Omega^{1 / 2}}^{\infty} \mathrm{d} \zeta \zeta^{2} \mathrm{e}^{-2 \zeta^{2}} Z(\mathrm{i} \zeta) \\
& \equiv 2 \Omega I_{1}+2 I_{2} \text {, }
\end{aligned}
$$

and

$$
2 \int_{0}^{\infty} \mathrm{d} \hat{v}_{\perp} \hat{v}_{\perp} \mathrm{e}^{-\hat{v}_{\perp}^{2}} \sqrt{\Omega-\hat{v}_{\perp}^{2} / 2 Z}\left(\sqrt{\Omega-\hat{v}_{\perp}^{2} / 2}\right)=-I_{2}
$$

Then

$$
1+\tau-\eta_{i} \Omega_{* i}=-\left\{\Omega-\Omega_{* i}\left[1-\frac{3}{2} \eta_{i}\left(1-\frac{4}{3} \Omega\right)\right]\right\} I_{1}-\eta_{i} \Omega_{* i} I_{2} .
$$

By writing the plasma dispersion function as an error function of imaginary argument, one sees that the integrals $I_{1}$ and $I_{2}$ can be performed analytically to obtain

$$
\begin{aligned}
1+\tau= & \left\{-\left(\Omega-\Omega_{* i}\right)-\eta_{i} \Omega_{* i}(1-2 \Omega)\right\} \pi \mathrm{e}^{-2 \Omega}\left[1-\operatorname{Erf} i^{2}(\sqrt{\Omega})-2 i \operatorname{Erf} i(\sqrt{\Omega})\right] \\
& +2 \eta_{i} \Omega_{* i} \sqrt{\pi \Omega} \mathrm{e}^{-\Omega}[\operatorname{Erf} i(\sqrt{\Omega})+i]
\end{aligned}
$$

whose imaginary part set to zero gives (F2).

\section{REFERENCES}

Bhattacharjee, A., Sedlak, J. E., Similon, P. L., Rosenbluth, M. N. \& Ross, D. W. 1983 Drift waves in a straight stellarator. Phys. Fluids 26 (4), 880-882.

Biglari, H., Diamond, P. H. \& Rosenbluth, M. N. 1989 Toroidal ion-pressure-gradient-driven drift instabilities and transport revisited. Phys. Fluids B 1 (1), 109-118.

Candy, J., Waltz, R. E. \& Rosenbluth, M. N. 2004 Smoothness of turbulent transport across a minimum-q surface. Phys. Plasmas 11 (5), 1879-1890.

Connor, J. W. \& Hastie, R. J. 2004 Microstability in tokamaks with low magnetic shear. Plasma Phys. Control. Fusion 46 (10), 1501.

Connor, J. W., HAStie, R. J. \& TAYlor, J. B. 1978 Shear, periodicity, and plasma ballooning modes. Phys. Rev. Lett. 40, 396.

CONNOR, J. W. \& TAYLOR, J. B. 1987 Ballooning modes or Fourier modes in a toroidal plasma? Phys. Fluids 30 (10), 3180-3185.

Coppi, B., Laval, G., Pellat, R. \& Rosenbluth, M. N. 1968 Collisionless microinstabilities in configurations with periodic magnetic curvature. Plasma Phys. 10 (1), 1.

Coppi, B., Rosenbluth, M. N. \& SAgdeev, R. Z. 1967 Instabilities due to temperature gradients in complex magnetic field configurations. Phys. Fluids 10 (3), 582.

Dewar, R. L. 1997 Spectrum of the ballooning Schrödinger equation. Plasma Phys. Control. Fusion 39 (3), 453.

Dickinson, D., RoACH, C. M., SkipP, J. M. \& Wilson, H. R. 2014 Structure of micro-instabilities in tokamak plasmas: stiff transport or plasma eruptions? Phys. Plasmas 21, 010702. 
Dimits, A. M., Bateman, G., Beer, M. A., Cohen, B. I., Dorland, W., Hammett, G. W., Kim, C., Kinsey, J. E., Kotschenreuther, M., Kritz, A. H. et al. 2000 Comparisons and physics basis of tokamak transport models and turbulence simulations. Phys. Plasmas 7 (3), 969-983.

Faber, B. J., Pueschel, M. J., Proll, J. H. E., Xanthopoulos, P., Terry, P. W., Hegna, C. C., Weir, G. M., Likin, K. M. \& TAlmadge, J. N. 2015 Gyrokinetic studies of trapped electron mode turbulence in the helically symmetric experiment stellarator. Phys. Plasmas 22 (7), 072305.

Fried, B. D. \& Conte, S. D. 1961 The Plasma Dispersion Function. Academic.

Geiger, J., Beidler, C. D., Feng, Y., MaAssberg, H., Marushchenko, N. B. \& Turkin, Y. 2015 Physics in the magnetic configuration space of W7-X. Plasma Phys. Control. Fusion 57 (1), 014004.

HAHM, T. S. \& TANG, W. M. 1989 Properties of ion temperature gradient drift instabilities in H-mode plasmas. Phys. Fluids B 1 (6), 1185-1192.

Hastie, R. J. \& Hesketh, K. W. 1981 Kinetic modifications to the MHD ballooning mode. Nucl. Fusion 21 (6), 651.

HILL, G. W. 1886 On the part of the motion of the lunar perigee which is a function of the mean motions of the sun and moon. Acta Math. 8 (1), 1-36.

Horton, W., ChOI, D. \& TANG, W. M. 1981 Toroidal drift modes driven by ion pressure gradients. Phys. Fluids 24 (6), 1077-1085.

Jenko, F., Dorland, W. \& Hammett, G. W. 2001 Critical gradient formula for toroidal electron temperature gradient modes. Phys. Plasmas 8 (9), 4096-4104.

Jenko, F., Dorland, W., Kotschenreuther, M. \& Rogers, B. N. 2000 Electron temperature gradient driven turbulence. Phys. Plasmas 7 (5), 1904-1910.

Kadomtsev, B. B.\& Pogutse, O. P. 1970 Reviews of Plasma Physics, vol. 5. Taylor and Francis.

Mantica, P., Strintzi, D., Tala, T., Giroud, C., Johnson, T., Leggate, H., Lerche, E., Loarer, T., Peeters, A. G., SAlmi, A. et al. 2009 Experimental study of the ion criticalgradient length and stiffness level and the impact of rotation in the JET tokamak. Phys. Rev. Lett. 102, 175002.

Owen, D. B. 1956 Tables for computing bivariate normal probabilities. Ann. Math. Statist. 27 (4), 1075-1090.

Plunk, G. G., Helander, P., Xanthopoulos, P. \& Connor, J. W. 2014 Collisionless microinstabilities in stellarators. III. The ion-temperature-gradient mode. Phys. Plasmas 21 (3), 032112 .

Proll, J. H. E., Xanthopoulos, P. \& Helander, P. 2013 Collisionless microinstabilities in stellarators. II. Numerical simulations. Phys. Plasmas 20 (12), 122506.

RomANelLI, F. 1989 Ion temperature-gradient-driven modes and anomalous ion transport in tokamaks. Phys. Fluids B 1 (5), 1018.

Romanelli, F. \& ZonCA, F. 1993 The radial structure of the ion-temperature-gradient-driven mode. Phys. Fluids B 5 (11), 4081-4089.

Rosenbluth, M. N. \& Longmire, C. L. 1956 Stability of plasmas confined by magnetic fields. Ann. Phys. 1 (2), 120.

Rudakov, L. I. \& SAGDEEV, R. Z. 1961 O neustoychivosti neodnorodnoy razrezhennoy plazmyi v silnom magnitnom pole. Dokl. Akad. Nauk CCCP 138, 581.

TAYlOR, J. B. \& HASTIE, R. J. 1968 Stability of general plasma equilibria - I formal theory. Plasma Phys. 10 (5), 479.

TAYlor, J. B. \& Wilson, H. R. 1996 Plasma rotation and toroidal drift modes. Plasma Phys. Control. Fusion 38 (11), 1999.

Terry, P., Anderson, W. \& Horton, W. 1982 Kinetic effects on the toroidal ion pressure gradient drift mode. Nucl. Fusion 22 (4), 487.

Tricomi, F. G. $1950 a$ Asymptotische eigenschaften der unvollständigen gammafunktion. Math. Z. 53 (2), 136-148.

Tricomi, F. G. $1950 \mathrm{~b}$ Sulla funzione gamma incompleta. Annali di Matematica Pura ed Applicata 31 (1), 263-279. 
Xanthopoulos, P., Cooper, W. A., Jenko, F., Turkin, Y., Runov, A. \& Geiger, J. 2009 A geometry interface for gyrokinetic microturbulence investigations in toroidal configurations. Phys. Plasmas 16 (8), 082303.

Zocco, A., Plunk, G. G., Xanthopoulos, P. \& Helander, P. 2016 Geometric stabilization of the electrostatic ion-temperature-gradient driven instability. I. Nearly axisymmetric systems. Phys. Plasmas 23 (8). 